\title{
12 Überzeugungen zur Mathematik und zum Erwerb mathematischen Wissens
}

Horst Biedermann, Christian Brühwiler, Fritz Oser, Benita Affolter und Andreas Bach

\begin{abstract}
Überzeugungen wird ein bedeutsamer Einfluss bezüglich der Zielvorstellungen, Wahrnehmungen und Deutungen unterrichtlicher Situationen beigemessen, wodurch sie grundsätzlich als bedeutsam hinsichtlich des berufsbezogenen Denkens und Handelns erachtet werden. Im Fokus der Ausbildung angehender Lehrpersonen interessiert daher, über welche Überzeugungen Studierende verfügen - so auch in TEDS-M in Bezug auf Überzeugungen zur Struktur der Mathematik, zum mathematischen Lehren und Lernen und zum Erwerb mathematischen Wissens. Die Ergebnisse zeigen, dass Studierende der Deutschschweiz (und auch in Deutschland) am Ende der Lehrerausbildung diesbezüglich weitgehend über dynamisch-konstruktivistisch-offene ÜberzeugungsSyndrome verfügen, wobei sich diese auch in internationaler Betrachtung als besonders ausgeprägt darstellen. Auch wird deutlich, dass derartige Überzeugungsmuster (zumindest tendenziell) in Beziehung zu intrinsischer Berufsmotivation (fachbezogener Motivation und Interesse an Lernenden) stehen. Ob derartig deutliche ÜberzeugungsSyndrome als vorteilhaft zu bewerten sind, lässt sich nicht abschliessend bewerten. Hier würde es sich lohnen, in weiteren Forschungsarbeiten der Frage nachzugehen, inwieweit es den Studierenden in ihrer Berufspraxis gelingt, situationsangepasst konstruktions- und transmissionsorientierte Lehr-Lernarrangements anzuwenden.
\end{abstract}

\subsection{Einleitung und Fragestellung}

Kompetentes Handeln basiert auf dem situationsbezogenen Zusammenspiel von kognitiven Leistungsdispositionen und motivationalen, volitionalen sowie sozialen Bereitschaften und Fähigkeiten, wie es Weinert $(1999,2001)$ in seiner vielbeachteten Bestimmung zum Ausdruck gebracht hat. Noch wenig explizit werden in dieser Bestimmung Überzeugungen mitgedacht. Diesbezüglich können nach Fenstermacher (1994) gerade das Wissen und die Überzeugungen als eng verschmolzen betrachtet werden, wobei ein zentrales Differenzierungsmerkmal darin besteht, dass sie unterschiedlichen Rechtfertigungsansprüchen 
unterliegen und daher unterschiedlichen epistemologischen Status aufweisen. So lassen sich Überzeugungen - in deutlicher Differenz zum Wissen - einzig mit dem individuellen Richtigkeitsglauben begründen, wodurch sie ,weder den Kriterien der Widerspruchsfreiheit noch den Anforderungen der argumentativen Rechtfertigung und der diskursiven Validierung zu genügen“ (Baumert \& Kunter, 2006, S. 497) haben. Bei Überzeugungen handelt es sich somit um etwas wie „Glaubensbestände" (Oser \& Blömeke, 2012, S. 416), wobei die enge Nähe zu Konzepten wie zu den ,subjektiven Theorien“ (z.B. Groeben, Wahl, Schlee \& Scheele, 1998), „Vorstellungen“ (z.B. Drechsel, 2001) oder „,pädagogische[n] Haltungen und Weltbilder“" (z.B. Dann, Müller-Fohrbrodt \& Cloetta, 1981) ersichtlich wird. Auch im englischen Sprachraum finden sich im Bemühen um Verdeutlichung je eigener Forschungsperspektiven derartige begriffliche Abgrenzungen vom breit anerkannten Begriff Beliefs (auf welchen aufgrund des inkonsistenten Begriffsverständnisses auch im deutschsprachigen Raum häufig zurückgegriffen wird) in Form von Cognitions, Conceptions, Views oder Orientations (z.B. Forgasz \& Leder, 2008; Lester, 2007; Reusser, Pauli \& Elmer, 2011; Schoenfeld, 1998; Wilson \& Cooney, 2002). Auch wenn Belief somit nach Pajares (1992) ein ,messy construct“ darstellt, so vereint sich in diesem Begriff doch die all den unterschiedlichen Bemühungen verbindende Vorstellung, ,wie etwas beschaffen ist oder wie etwas funktioniert, mit dem Anspruch auf Geltung für das Handeln“ (Oser \& Blömeke, 2012, S. 415).

Auf den Lehrberuf bezogen kann unter berufsbezogenen Überzeugungen bzw. Beliefs , affektiv aufgeladene, eine Bewertungskomponente beinhaltende Vorstellungen über das Wesen und die Natur von Lehr-Lernprozessen, Lerninhalten, die Identität und Rolle von Lernenden und Lehrenden (sich selbst) sowie den institutionellen und gesellschaftlichen Kontext von Bildung und Erziehung, welche für wahr oder wertvoll gehalten werden und ihrem berufsbezogenen Denken und Handeln Struktur, Halt, Sicherheit und Orientierung geben“ (Reusser et al., 2011, S. 478) verstanden werden. Überzeugungen wird somit ein signifikanter Einfluss auf den Bewertungsmassstab des eigenen professionellen Handelns zugesprochen. Deshalb sind sie als bedeutsam hinsichtlich der Zielvorstellungen, der Wahrnehmungen und Deutungen unterrichtlicher Situationen, der Erwartungen gegenüber Schülerinnen und Schülern und somit ganz allgemein bezüglich des professionellen Handelns von Lehrpersonen zu betrachten (z.B. Bråten, Britt, Strømsø \& Rouet, 2011; Goldin, Rösken \& Törner, 2009; Hofer, 2001; König, 2012; Reusser et al., 2011; Richardson, 1996) - wobei diesbezügliche datenbasierte Nachweise bereits mehrmals vorgelegt werden konnten (z.B. Peterson, Fennema, Carpenter \& Loef, 1989; Niederhauser \& Stoddart, 2001; Staub \& Stern, 2002; Stipek, Givvin, Salmon \& MacGyvers, 2001).

Im Bemühen um Strukturierung derartiger „Glaubensbestände“ bzw. Überzeugungen von (im Bereich der Forschung zumeist bezogen auf Mathematik-)Lehrpersonen hat sich in der Literatur folgende Einteilung weitgehend durchgesetzt: (a) domänenspezifische epistemologische Überzeugungen (zu 
Inhalten sowie zum Wesen und zur Struktur von Fächern), (b) allgemein epistemologische Überzeugungen über die Prozesse des Lehrens und Lernens, (c) personengebundene Überzeugungen (zu Akteuren des bildungsbezogenen Handelns im Schulkontext; auch zu professionsbezogenen Selbstwahrnehmungen) sowie (d) kontextbezogene Überzeugungen (zu Merkmalen von Schule und Gesellschaft) (Blömeke, Müller, Felbrich \& Kaiser, 2008; Hofer \& Pintrich, 2002; Op't Eynde, De Corte \& Verschaffel, 2002; Reusser et al., 2011). In Anlehnung an diese Gliederung werden in TEDS-M folgende drei Überzeugungsdimensionen betrachtet (nicht berücksichtigt sind in dieser Studie die kontextbezogenen Überzeugungen [Punkt d]):

- Überzeugungen zur Struktur von Mathematik: In Anlehnung an Grigutsch, Raatz und Törner (1998) wurde eine zweifaktorielle Operationalisierung in Differenzierung einer statischen und dynamischen Perspektive auf die Mathematik vorgenommen. Mit der statischen Perspektive verbindet sich die Vorstellung von Mathematik als ein Konglomerat von Definitionen, Formeln und mathematischen Fakten. Die dynamische Perspektive betont demgegenüber den prozesshaften und anwendungsbezogenen Charakter der Mathematik.

- Personengebundene Überzeugungen zum mathematischen Lernen: Mit der Operationalisierung von Mathematik bzw. mathematischen Könnens als eine angeborene Fähigkeit soll erhellt werden, inwiefern die angehenden Lehrpersonen einen Zugang zu Mathematik als allen Menschen offen oder aber von Natur aus vorgegeben bewerten.

- Überzeugungen zum Erwerb mathematischen Wissens: Die Operationalisierung der Vorstellungen zum mathematischen Lehren und Lernen erfolgte wiederum zweifaktoriell. Auf der einen Seite wird durch die transmissionsorientierte Perspektive die Vorstellung eines gerichteten Vermittlungsprozesses fachbezogener Inhalte von der Lehrperson auf die Schülerinnen und Schüler zum Ausdruck gebracht. Demgegenüber zeigt die konstruktivistische Perspektive einen stärker selbstgesteuerten aktiven Konstruktionsprozess der Schülerinnen und Schüler auf.

In diesem Beitrag wird somit folgenden Fragestellungen nachgegangen:

1. Über welche Überzeugungen zur Struktur der Mathematik, zum mathematischen Lernen und zum Erwerb mathematischen Wissens verfügen angehende Lehrpersonen der Deutschschweiz?

2. Lassen sich Unterschiede in den Überzeugungen zur Mathematik zwischen den Lehramtsstudierenden der Deutschschweiz und den TEDS-M Ländern feststellen?

3. Unterscheiden sich Überzeugungen zur Mathematik angehender Lehrpersonen in unterschiedlichen Ausbildungsgängen der Deutschschweiz? 
4. Bestehen Zusammenhänge zwischen motivationalen (Berufs-)Orientierungen und Überzeugungen zur Mathematik?

Im Übergang zum 21. Jahrhundert wurden verstärkt konstruktionsorientierte Lehr- Lernmethoden für erfolgreiche Lernentwicklungen verantwortlich gemacht, wodurch auch in den Lehrerbildungen der Schweiz und von Deutschland Lehrkonzepte entwickelt und umgesetzt wurden, die den Lehr-Lernprozess als subjektiven Konstruktionsprozess betonen und entsprechende Überzeugungen bei Studierenden verstärken sollen (Reusser et al., 2011). Dementsprechend ist zu vermuten, dass sich diese Entwicklung auch in den Ergebnissen dieser Studie niederschlägt. Konkret ist davon auszugehen, dass bedeutsam stärker konstruktions- als transmissionsorientierte Überzeugungen wirksamen Lernens zum Ausdruck gebracht werden. Mit der konstruktivistischen Sichtweise verbindet sich auch stärker eine dynamische als statische Perspektive zur Struktur von Mathematik sowie eine offene und somit nicht naturgegebene Zugangsmöglichkeit zum mathematischen Können. Im Einklang mit der hier vermuteten konsistenten Überzeugungsrichtung der drei Dimensionen konnte in jüngerer Vergangenheit aufgezeigt werden, dass sich zwischen einzelnen (Teil-)Kategorien deutliche Beziehungen zeigen, so dass diesbezüglich von „Überzeugungs-Syndromen“ (Blömeke et al., 2008, S. 240) oder „Präferenzmustern“ (Baumert et al., 2004, S. 333) ausgegangen werden kann.

Diese Erwartung von deutlich hervortretenden dynamisch-konstruktivistisch-offenen Überzeugungen gegenüber Mathematik sowie mathematischem Lehren und Lernen bedeutet jedoch keineswegs, dass damit verbunden statisch-transmissive Sichtweisen ausgeschlossen werden müssen oder auch ein deutliches Überwiegen des erwarteten Präferenzmusters wünschenswert wäre. Führt man sich vor Augen, dass sich wirksamer Unterricht wohl gerade durch den adäquaten Einsatz transmissions- und konstruktionsbasierter Methoden auszeichnet (z.B. derart als dass in Form der Transmission auch einmal ein Input gestaltet wird, worauf basierend Vertiefungen und Anwendungen konstruktionsorientiert angegangen werden), so kann auch eine diesbezügliche Ausbalancierung der Überzeugungen als wünschenswert betrachtet werden. Aufgrund der oben erwähnten Fokussierung konstruktionsorientierter LehrLernmethoden ist für die Deutschschweiz (und auch für Deutschland) jedoch eine derartige Ausbalancierung nicht zu erwarten. Vielmehr ist anzunehmen, dass sich diese Ausbildungskonzeption auch in den internationalen Ergebnissen niederschlägt, so dass die Deutschschweiz in Bezug auf dynamisch-konstruktivistisch-offene Überzeugungen in oberen Bereichen der Ländervergleiche und hinsichtlich statisch-transmissiven Überzeugungen in unteren Bereichen liegen wird. Da davon auszugehen ist, dass alle Lehrerbildungsinstitutionen der Deutschschweiz konstruktionsorientierte Lehr-Lernmethoden anwenden, wird vermutet, dass zwischen den Ausbildungsgängen keine bedeutsamen Unterschiede bestehen. 
Obschon unbestritten ist, dass den berufsbezogenen Überzeugungen von Lehrpersonen handlungsleitender Charakter für das Unterrichtshandeln zukommt, ist erst wenig über die Wirkungsweise bekannt (z.B. Müller, Felbrich \& Blömeke, 2008; Reusser et al., 2011). Vor diesem Hintergrund interessieren auch Zusammenhänge zwischen Überzeugungen im Bereich Mathematik und motivationalen Orientierungen angehender Lehrpersonen (zu motivationalen Orientierungen siehe Kapitel 4 in diesem Band). Eine Besonderheit des Lehrberufs und der Lehrerausbildung besteht darin, dass Studierende bereits beim Eintritt in die Ausbildung über vieljährige Erfahrungen im Berufsfeld Schule und Unterricht verfügen. Damit verbunden besitzen sie gefestigte Überzeugungen zum „guten“ bzw. „wirksamen“ Lehren und Lernen, welche sich insbesondere aus den eigenen Schulerfahrungen speisen was Lortie (1975) als „Apprenticeship of Observation“ bezeichnet hat. In den 1970er Jahren war diesbezüglich festzustellen, dass Studierende mit eher konservativen Überzeugungen in die Ausbildung eintreten und sich diese dann während der Ausbildung zu liberaleren Überzeugungen entwickeln, bevor sie in der Berufseingangsphase wiederum auf den Stand zu Beginn der Ausbildung „,zurückfallen“, was unter dem Schlagwort „Konstanzer-Wanne“ diskutiert wurde (vgl. z.B. Koch, 1972; Müller-Fohrbrodt, Cloetta \& Dann, 1978). Demgegenüber zeigen jüngste Ergebnisse, dass bereits Studienbeginnende stärker über dynamisch-konstruktivistische als über statisch-transmissionsorientierte Überzeugungen verfügen (Biedermann, Brühwiler \& Steinmann, 2012). Diesbezüglich ist zu vermuten, dass sich die konstruktionsorientierten Lehr-Lernmodelle der Lehrerausbildung bereits auf den Unterricht der (Jung-) Lehrpersonen ausgewirkt haben, so dass Schülerinnen und Schüler ihre Alltagserfahrungen auch auf derartigen Lehr-Lernarrangements aufbauen. Somit ist anzunehmen, dass eine hohe fachbezogene Motivation der Studierenden mit einer dynamischen Perspektive zur Struktur von Mathematik und mit einer höheren Konstruktionsorientierung einhergeht.

Der Beitrag ist so gegliedert, dass zuerst die Ergebnisse der Studierenden der Deutschschweiz im internationalen und nationalen Vergleich betrachtet werden. Daran anschliessend werden Beziehungen zwischen den Überzeugungen und motivationalen Orientierungen analysiert, bevor abschliessend ein Fazit gezogen wird.

\subsection{Ergebnisse - Überzeugungen zur Mathematik und zum mathematischen Lehren und Lernen}

Im Folgenden werden die Ergebnisse der Studierenden der Deutschschweiz sowohl im internationalen als auch im nationalen Vergleich betrachtet. Dabei interessiert auf internationaler Ebene insbesondere der Vergleich mit den Län- 
dern Deutschland, Polen, Russland, Singapur, Spanien, Taiwan und USA, da diese kulturell der Deutschschweiz (noch) am nächsten stehen und/oder die besten Ergebnisse in den TEDS-M-Tests zum Mathematikwissen und zur Mathematikdidaktik (vgl. Kapitel 10 und 11 in diesem Band) erreichten. In den Abbildungen sind jeweils einerseits die Ergebnisse der Länder insgesamt und andererseits die Ausbildungsgänge der Deutschschweiz nach Ausbildungstypen (in Differenzierung nach Untere Primarstufe [Klassen 1-4], Primarstufe [Klassen 1-6] und Sekundarstufe I) dargestellt. Dieser Blick auf die einzelnen Ausbildungstypen wird darüber hinaus verschiedentlich auch auf Länderebene vollzogen bzw. es werden die diesbezüglichen Ergebnisse beschrieben, wobei an den jeweiligen Stellen darauf aufmerksam gemacht wird.

\subsection{1 Überzeugungen zur Struktur der Mathematik: statische und dynamische Perspektiven}

Zur Erfassung der Überzeugungen zur Struktur der Mathematik wurden auf der Basis von elf Items zwei Skalen gebildet, die zwischen einer statischen und dynamischen Perspektive differenzieren. Während die statische Sichtweise die Bedeutung von mathematischen Fakten, Formeln und Definitionen betont, werden mit der dynamischen Perspektive Überzeugungen erfasst, die den prozesshaften und anwendungsbezogenen Charakter des Fachs hervorheben.

\section{Statische Perspektive}

Die in Tabelle 12.1 aufgeführten Items zur Erfassung von Überzeugungen zur Struktur der Mathematik in einer statischen Perspektive betonen einerseits die Bedeutung von mathematischen Fakten, Formeln und Regeln (z.B. „Mathematik ist eine Sammlung von Regeln und Verfahren, die beschreiben, wie eine Aufgabe zu lösen ist"). Andererseits fokussieren sie Voraussetzungen (z.B. „Um ein mathematisches Problem zu lösen, muss man das korrekte Verfahren kennen, ansonsten ist man aufgeschmissen“) und Bedingungen (z.B. „Wer sich mit Mathematik beschäftigt, muss viel üben, Verfahren korrekt anwenden und über verschiedene Problemlösestrategien verfügen"), die vorhanden bzw. gegeben sein müssen, um erfolgreich im Fach Mathematik zu sein. Die Skala umfasst sechs Items, die sechsstufig („Stimme überhaupt nicht zu“ bis „Stimme völlig zu“") beantwortet wurden. 
Tabelle 12.1: Statische Perspektive zur Struktur der Mathematik Items und Antwortmöglichkeiten

Wie sehr stimmen Sie den folgenden Aussagen über das Wesen der Mathematik zu?

Items

a) Mathematik ist eine Sammlung von Regeln und Verfahren, die beschreiben, wie eine Aufgabe zu lösen ist.

b) Mathematik beinhaltet das Erinnern und Anwenden von Definitionen, Formeln, mathematischen Fakten und Verfahrensweisen.

c) Um ein mathematisches Problem zu lösen, muss man das korrekte Verfahren kennen, ansonsten ist man aufgeschmissen.

d) Grundlegend für die Mathematik sind logische Strenge und Eindeutigkeit.

e) Wer sich mit Mathematik beschäftigt, muss viel üben, Verfahren korrekt anwenden und über verschiedene Problemlösestrategien verfügen.

f) Mathematik bedeutet lernen, erinnern und anwenden.

Antwortmöglichkeiten: Stimme überhaupt nicht zu - Stimme nicht zu-Stimme eher nicht zu - Stimme eher zu -Stimme zu - Stimme völlig zu; Cronbach Alpha (CHE): .71 (Prim); .70 (Sek)

Wie aus Abbildung 12.1 ersichtlich wird, liegt die Deutschschweiz hinsichtlich einer statischen Perspektive zur Struktur von Mathematik mit einem Mittelwert von $9.99^{1}$ für die Primarstufe bzw. 9.86 für die Sekundarstufe nahe beim neutralen Skalen-Mittelpunkt von 10 und belegt im internationalen Ländervergleich den untersten bzw. vorletzten Platz. Zusammen mit Deutschland unterscheidet sich die Deutschschweiz dann auch sowohl auf der Primar- als auch der Sekundarstufe von allen anderen Ländern statistisch signifikant negativ. Studierende in der Deutschschweiz sind demnach im Mittel neutral bis leicht ablehnend davon überzeugt, dass die Mathematik einer statischen Struktur unterliegt. Die diesbezüglich höchsten Zustimmungen lassen sich auf der Primarstufe für Singapur (11.05), die USA (11.01) und Polen (10.91) feststellen. Auf der Sekundarstufe erlangt die USA den höchsten Mittelwert (11.04), gefolgt von Singapur (10.92) und Taiwan (10.81). Die Streuungen fallen im Ländervergleich für die Deutschschweiz gering aus $(\mathrm{SD}=.71$ für die Primarstufe; $\mathrm{SD}=.69$ für die Sekundarstufe), dies z.B. in deutlichem Unterschied zu Polen und den USA, die mit Standardabweichungen von 1.06 bis 1.31 in Bezug auf beide Zielstufen die höchsten Werte aufweisen.

In Betrachtung der Ausbildungstypen bestätigen sich die oben berichteten typenübergreifenden Länderresultate durchwegs. Sowohl für die Untere Primarstufe (1-4) mit 10.10, für die Primarstufe (1-6) mit 9.98 als auch für die

1 Es gilt zu beachten, dass die in Tabelle 12.1 vorgestellte sechsstufige Likert-Skala und die in diesem Kapitel noch folgenden Skalen im Rahmen der von der IEA durchgeführten Skalenanalysen (IRT-Modellierungen) in je standardisierte Skalen mit einem neutralen Mittelpunkt von 10 (was auf der sechsstufigen Likert-Skala mit Werten von 1 bis 6 dem Punkt 3.5 entsprechen würde) überführt wurden. 
Sekundarstufe I mit 9.86 liegen die Mittelwerte der Deutschschweiz am unteren Ende der Vergleiche - und dies auch jeweils in statistischer Signifikanz zu den anderen Ländern mit Ausnahme zu Deutschland, dessen Ergebnisse stets (sofern die entsprechenden Ausbildungstypen auch vorhanden sind) im Bereich der Deutschschweiz liegen.

Richtet man den Blick auf die einzelnen Ausbildungsgänge der Deutschschweiz, so wird augenfällig, dass die Mittelwerte zwar nicht so ausgeprägt wie in internationaler Betrachtung variieren, sich aber dennoch Unterschiede zeigen. Die Mittelwerte reichen für die Untere Primarstufe (1-4) von 9.70 bis 10.38, für die Primarstufe (1-6) von 9.37 bis 10.35 und für die Sekundarstufe I von 9.40 bis 10.20 (Abbildung 12.1). Eine statistisch bedeutsame Differenz lässt sich dabei aber lediglich für die Ausbildungsgänge an den Gegenpolen der Listen ausweisen. So unterscheiden sich auf der Unteren Primarstufe (1-4) die Ausbildungsgänge U1 und U5 von U3 und U4, auf der Primarstufe (1-6) primär $\mathrm{P} 3$ positiv und P14 negativ von beinahe allen anderen Studiengängen und auf der Sekundarstufe I S4 positiv von S7, S6 und S2 sowie S2 negativ von S4, S3 und S5. Ebenfalls insgesamt homogener als im internationalen Vergleich zeigen sich die Streuungen für die einzelnen Ausbildungsgänge. 
Abbildung 12.1: Überzeugung zur Struktur der Mathematik: statische Perspektive - internationaler Ländervergleich und Institutionen der Deutschschweiz

$\begin{array}{lll} & & \text { Überzeugung zur Struktur der Mathematik: }\end{array}$

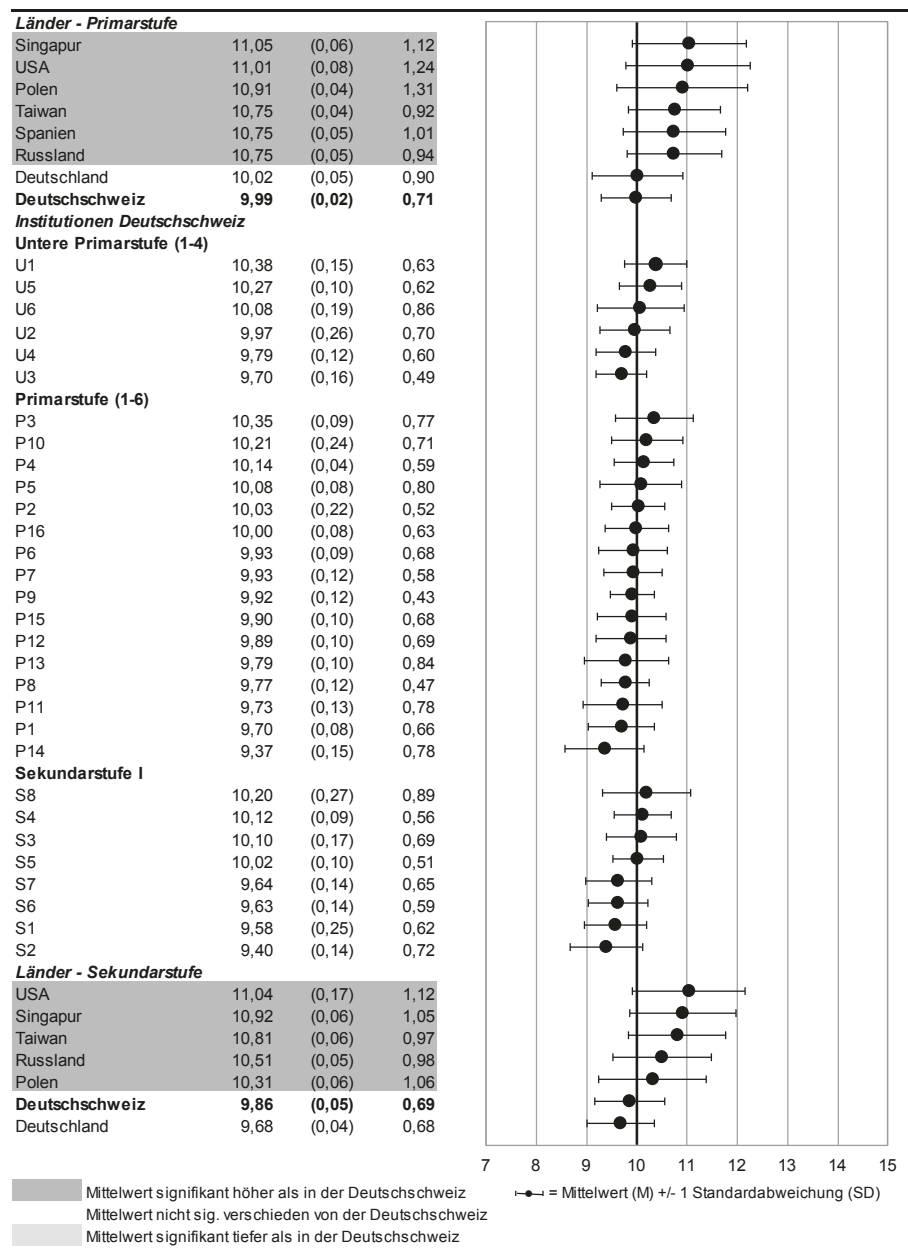

Anmerkung: $\mathrm{M}=$ Mittelwert, $\mathrm{SE}=$ Standardfehler, $\mathrm{SD}=$ Standardabweichung; Sekundarstufe $\mathrm{I}=$ Ausbildungsprogramme mit Unterrichtsberechtigung bis 9. Klassenstufe, Sekundarstufe= Ausbildungsprogramme mit Unterrichtsberechtigung auf Sekundarstufe I und II, d.h. bis Klassenstufe 11/12 (vgl. Kapitel 2 in diesem Band); Abbildung=Mittelwert +/- 1 SD, der theoretische Skalen-Mittelpunkt liegt bei 10, d.h. ein Wert von grösser 10 bedeutet eine Zustimmung und ein Wert von kleiner 10 eine Ablehnung bezüglich des gemessenen Konstrukts. 


\section{Dynamische Perspektive}

Die Überzeugung zu Mathematik als dynamische Struktur basiert auf fünf Items, die ebenfalls sechsstufig (,Stimme überhaupt nicht zu“ bis „Stimme völlig zu“) beantwortet wurden (Tabelle 12.2). Die Aussagen fokussieren einerseits das der Mathematik zugrunde liegende Potential selbstentdeckenden und -gesteuerten Lernens (z.B. „In der Mathematik kann man viele Dinge selber entdecken und ausprobieren") und andererseits den fachbezogenen Anwendungsbezug (z.B. „Die Mathematik hilft, Probleme und Aufgaben im täglichen Leben zu lösen“").

Tabelle 12.2: Dynamische Perspektive zur Struktur der Mathematik Items und Antwortmöglichkeiten

Wie sehr stimmen Sie den folgenden Aussagen über das Wesen der Mathematik zu?

Items

a) In der Mathematik kann man viele Dinge selber entdecken und ausprobieren.

b) Wenn man sich mit mathematischen Problemen auseinandersetzt, kann man neue Dinge (wie Verbindungen, Regeln oder Konzepte) entdecken.

c) Mathematische Aufgaben und Probleme können auf verschiedene Weise korrekt gelöst werden.

d) Viele Aspekte der Mathematik haben eine praktische Bedeutung.

e) Die Mathematik hilft, Probleme und Aufgaben im täglichen Leben zu lösen.

Antwortmöglichkeiten: Stimme überhaupt nicht zu - Stimme nicht zu - Stimme eher nicht zu

- Stimme eher zu - Stimme zu - Stimme völlig zu; Cronbach Alpha (CHE):.77 (Prim); .76 (Sek)

Im Vergleich zur statischen Perspektive auf die Mathematik stimmen die Studierenden der Deutschschweiz der dynamischen Perspektive deutlich positiver zu. Die Mittelwerte liegen mit 11.32 für die Primarstufe und mit 11.73 für die Sekundarstufe klar über dem Skalen-Mittelpunkt von 10 und damit im zustimmenden Bereich (Abbildung 12.2). Dennoch befindet sich die Deutschschweiz im internationalen Ländervergleich lediglich im mittleren (Primarstufe) oder sogar untersten Bereich (Sekundarstufe). Auf der Primarstufe weist die USA den höchsten Mittelwert auf (12.18), gefolgt von Singapur (11.99), Taiwan (11.94) und Spanien (11.91). Am unteren Ende der Liste finden sich zusammen mit der Deutschschweiz Deutschland (11.28), Polen (11.26) und Russland (11.20). Auf der Sekundarstufe hebt sich die USA (12.50) positiv von der Deutschschweiz ab. Zu allen anderen Ländern ergeben sich keine statistisch signifikanten Differenzen (11.42 bis 12.08). Die Standardabweichungen fallen für die Deutschschweiz im internationalen Ländervergleich (mit Ausnahme von Russland auf der Primarstufe) am geringsten aus (je $\mathrm{SD}=1.24$ für die Primar- und Sekundarstufe).

In Betrachtung der Ergebnisse nach den Ausbildungstypen wird ersichtlich, dass die - zwar immer noch positiven, aber dennoch - geringsten Zustim- 
mungen zur dynamischen Perspektive von Mathematik auf der Unteren Primarstufe (1-4) zu finden sind. Hier steht die Deutschschweiz mit 11.25 an oberster Stelle des Ländervergleichs, wobei sich die Unterschiede zu den anderen Ländern im Zufallsbereich bewegen (mit Ausnahme zu - dem hier nicht primär interessierenden - Georgien, das mit 10.25 bedeutsam abfällt). Obwohl die Schweizer Studierenden der Primarstufe (1-6) mit einem Mittelwert von 11.33 den Durchschnitt ihrer nationalen Kolleginnen und Kollegen der Unteren Primarstufe (1-4) gering übertreffen, befinden sie sich am Ende des Ländervergleichs - und dies zu allen anderen Ländern (11.86 bis 13.25) in bedeutsamer Grösse. Die höchste Zustimmung innerhalb der Deutschschweiz findet sich mit einem Mittelwert von 11.73 für die Studierenden der Sekundarstufe. Von den Vergleichsländern unterscheiden sich nur die USA (12.36) statistisch signifikant (positiv) von der Deutschschweiz. Hinsichtlich der Streuungen sticht schliesslich hervor, dass die Ergebnisse der Deutschschweiz für alle drei Ausbildungstypen am geringsten ausfallen. 
Abbildung 12.2: Überzeugung zur Struktur der Mathematik:

dynamische Perspektive - internationaler Ländervergleich und Institutionen der Deutschschweiz

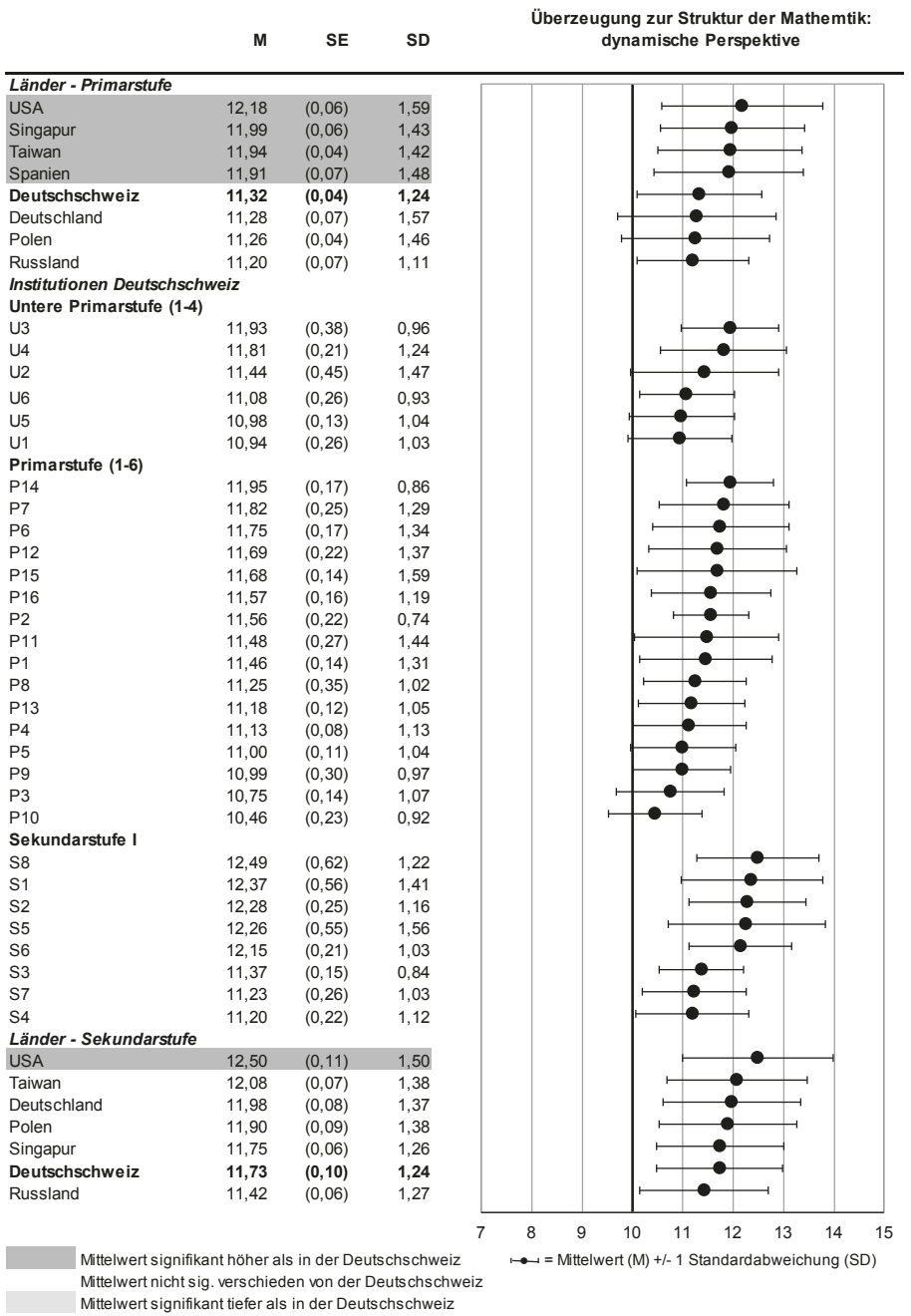

Anmerkung: $\mathrm{M}=$ Mittelwert, $\mathrm{SE}=$ Standardfehler, $\mathrm{SD}=$ Standardabweichung;

Sekundarstufe I=Ausbildungsprogramme mit Unterrichtsberechtigung bis 9. Klassenstufe, Sekundarstufe=Ausbildungsprogramme mit Unterrichtsberechtigung auf Sekundarstufe I und II, d.h. bis Klassenstufe 11/12 (vgl. Kapitel 2 in diesem Band); Abbildung=Mittelwert +/- 1 SD, der theoretische Skalen-Mittelpunkt liegt bei 10, d.h. ein Wert von grösser 10 bedeutet eine Zustimmung und ein Wert von kleiner 10 eine Ablehnung bezüglich des gemessenen Konstrukts. 
Auf Ebene der Ausbildungsgänge der Deutschschweiz offenbaren sich teilweise deutliche Mittelwertunterschiede (Abbildung 12.2). Diese fallen auf der Unteren Primarstufe (1-4) - mit Ausnahme zwischen U4 und U5 - jedoch nicht statistisch bedeutsam aus. Auf der Primarstufe (1-6) heben sich insbesondere P14 positiv (von P13, P4, P5, P3 und P10) sowie P3 und P10 negativ (von P14, P7, P6, P12, P15, P16, P2 und P1) ab. Und auf der Sekundarstufe I zeigt sich eine statistisch bedeutsame Differenz zwischen S2 sowie S6 und S3, S7 sowie S4. Sowohl die Standardfehler als auch die Standardabweichungen fallen für die Ausbildungsgänge teilweise gross aus, weshalb auch die Unterschiede nur in den dargestellten Fällen statistisch signifikant sind.

\subsubsection{Mathematik als angeborene Fähigkeit}

Die Überzeugung, dass mathematisches Können (primär) eine angeborene Fähigkeit darstelle, wurde mit einer acht Items umfassenden Skala erhoben, die drei Teilbereiche fokussiert: Die Studierenden wurden gebeten anzugeben, inwiefern sie denken, (1) dass es sich bei mathematischen Fähigkeiten um eine genetisch determinierte, zeitlich weitgehend stabile Disposition handelt (z.B. „Mathematik ist ein Fach, in dem Begabung viel bedeutender ist als Anstrengung"), (2) ob gruppenspezifische Unterschiede hinsichtlich mathematischer Fähigkeiten existieren (z.B. „Manche ethnischen Gruppen sind in Mathematik besser als andere") sowie (3) welche Konsequenzen sich aus dem mathematischen Fähigkeitsniveau ergeben (z.B. „Da ältere Schülerinnen und Schüler abstrakt denken können, ist die Verwendung von konkreten Modellen und anderen visuellen Hilfsmitteln weniger wichtig“) (Tabelle 12.3). Die Aussagen konnten durch sechs vorgegebene Möglichkeiten bewertet werden, von „Stimme überhaupt nicht zu“ bis „Stimme völlig zu“. 
Tabelle 12.3: Überzeugung zu Mathematik als angeborene Fähigkeit Items und Antwortmöglichkeiten

Wie sehr stimmen Sie den folgenden Aussagen zur Schülerleistung in Mathematik in der Primar-/Sekundarstufe I zu?

Items

a) Da ältere Schülerinnen und Schüler abstrakt denken können, ist die Verwendung von konkreten Modellen und anderen visuellen Hilfsmitteln weniger wichtig.

b) Um gut in Mathematik zu sein, muss man eine Art "mathematisches Gehirn" haben.

c) Mathematik ist ein Fach, in dem Begabung viel bedeutender ist als Anstrengung.

d) Nur die begabteren Schülerinnen und Schüler können mehrschrittige Problemlöseaufgaben bewältigen.

e) Im Allgemeinen sind Knaben von Natur aus besser in Mathematik als Mädchen.

f) Mathematische Fähigkeiten sind etwas, was sich über das Leben hinweg wenig verändert.

g) Manche Menschen sind gut in Mathematik und manche nicht.

h) Manche ethnischen Gruppen sind in Mathematik besser als andere.

Antwortmöglichkeiten: Stimme überhaupt nicht zu - Stimme nicht zu-Stimme eher nicht zu

- Stimme eher zu - Stimme zu - Stimme völlig zu; Cronbach Alpha (CHE):.72 (Prim); .72 (Sek)

Mit einem Mittelwert von 9.12 für die Primarstufe und 9.17 für die Sekundarstufe liegt die Deutschschweiz deutlich unter dem neutralen Skalen-Mittelpunkt von 10, wodurch sie sich auch im internationalen Ländervergleich (zusammen mit den USA und [jedoch nur auf Sekundarstufe] Deutschland) am Ende der Liste befindet (Abbildung 12.3). Studierende in der Deutschschweiz sind demzufolge mehrheitlich stärker davon überzeugt, dass es sich bei Mathematik um eine erlernbare als um eine angeborene Materie handelt. An der Spitze des internationalen Vergleichs liegt Russland (10.13 auf der Primarstufe und 10.08 auf der Sekundarstufe), gefolgt von Polen (10.07 und 9.89) und Taiwan (9.78 und 9.83). Diese Ergebnisse lassen erkennen, dass auch die international höchsten Mittelwerte nicht im Bereich der deutlichen Zustimmung angesiedelt sind, sondern um den neutralen Skalen-Mittelpunkt streuen. Bezüglich der nationalen Ergebnisstreuungen ist bemerkenswert, dass diese bei höheren Mittelwerten geringer als bei tieferen ausfallen. Höhere Mittelwerte basieren dementsprechend nicht nur auf deutlicherer Zustimmung, sondern auch auf homogeneren Bewertungen der Studierenden.

In Betrachtung der Länderergebnisse nach Ausbildungstypen bestätigen sich die dargelegten Resultate weitgehend. Die Deutschschweiz findet sich sowohl auf der Unteren Primarstufe (1-4; M=9.13), auf der Primarstufe (1-6; 9.11) als auch auf der Sekundarstufe I (9.17) am unteren Ende des Vergleichs, wobei für alle drei Ausbildungstypen ablehnende Überzeugungen zum Ausdruck gebracht werden. 
Eher geringe Unterschiede ergeben sich zwischen den Ausbildungsgängen der Deutschschweiz (Abbildung 12.3). Für die Untere Primarstufe (1-4) offenbaren sich keine statistisch signifikanten Differenzen. Auf der Primarstufe (1-6) zeigen sich die bedeutsamen Unterschiede nur gerade zwischen den Ausbildungsgängen an den jeweiligen Polen der Rangliste (so insbesondere zwischen einerseits P2, P6 und P9 und andererseits P4, P14 und P12). Und auf der Sekundarstufe I unterscheiden sich die Ausbildungsgänge S4 und S5 von S7 und S2, wobei S4 auch noch von S6 differenziert. Augenfällig ist schliesslich auch hier, dass die Streuungen bei höheren Mittelwerten zumeist geringer als bei tieferen ausfallen, wodurch höhere Mittelwerte insbesondere durch homogenere Überzeugungen der Studierenden erklärt werden können. 
Abbildung 12.3: Überzeugung zu Mathematik als angeborene

Fähigkeit - internationaler Ländervergleich und Institutionen der Deutschschweiz

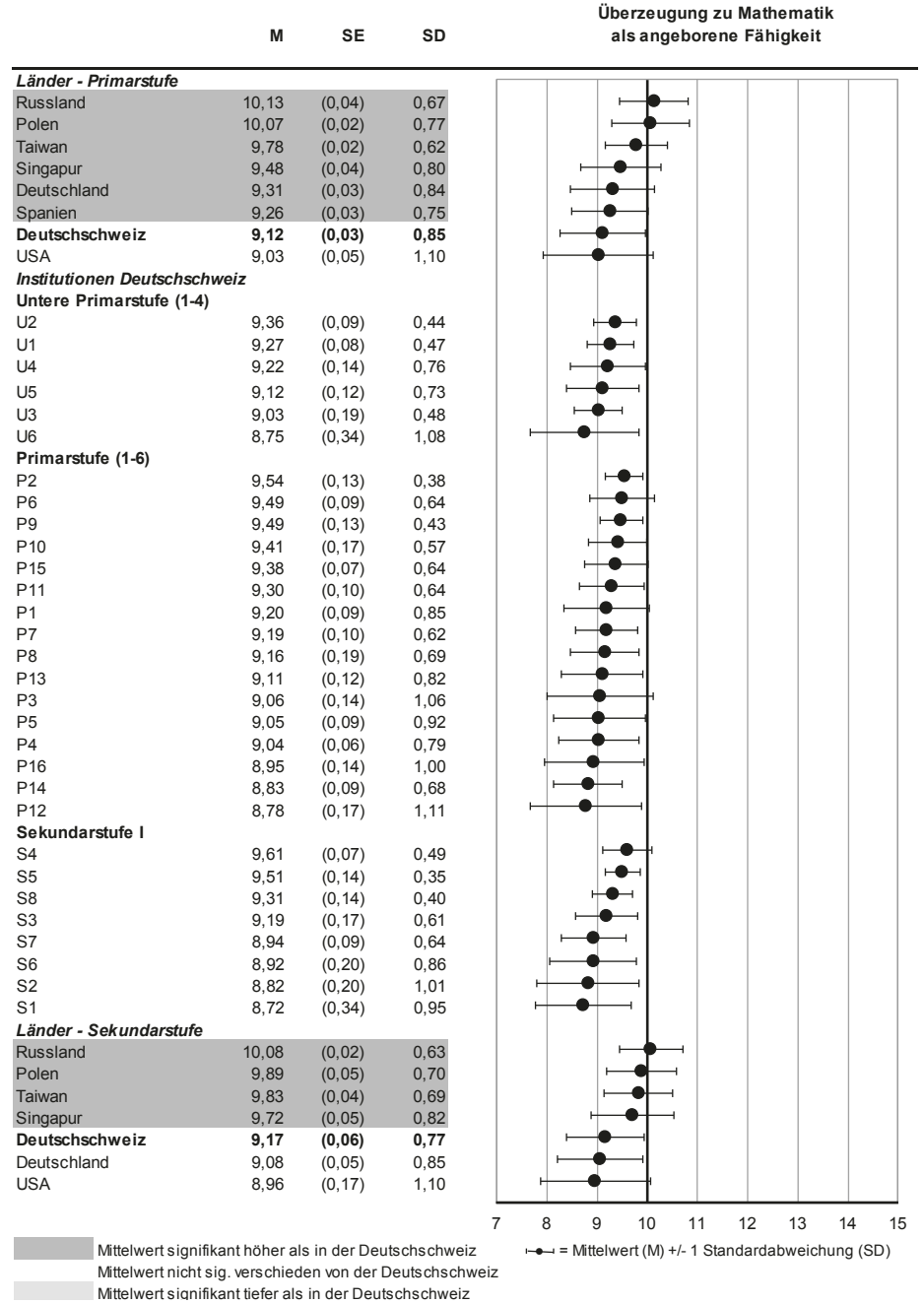

Anmerkung: $\mathrm{M}=$ Mittelwert, $\mathrm{SE}=$ Standardfehler, $\mathrm{SD}=$ Standardabweichung;

Sekundarstufe $\mathrm{I}=$ Ausbildungsprogramme mit Unterrichtsberechtigung bis 9 . Klassenstufe, Sekundarstufe=Ausbildungsprogramme mit Unterrichtsberechtigung auf Sekundarstufe I und II, d.h. bis Klassenstufe 11/12 (vgl. Kapitel 2 in diesem Band); Abbildung=Mittelwert +/- 1 SD, der theoretische Skalen-Mittelpunkt liegt bei 10, d.h. ein Wert von grösser 10 bedeutet eine Zustimmung und ein Wert von kleiner 10 eine Ablehnung bezüglich des gemessenen Konstrukts. 


\subsection{3 Überzeugungen zum Erwerb mathematischen Wissens}

Die Überzeugungen zum mathematischen Lehren und Lernen bzw. zum Erwerb mathematischen Wissens wurden nach den beiden Sichtweisen der Transmission und Konstruktion erhoben. Damit folgt TEDS-M einer Unterscheidung, wie sie sich in den letzten Jahren weitgehend durchgesetzt hat (angestossen durch Arbeiten wie beispielsweise von Calderhead [1996] und Staub und Stern [2002]). Die Transmissionsorientierung steht dabei für die Überzeugung, dass wirksame Lehr-Lernprozesse durch Wissensvermittlung der Lehrperson an die Schülerinnen und Schüler realisiert werden können (z.B. durch Frontalunterricht anhand von Lehrvorträgen). Die konstruktivistische Sichtweise geht demgegenüber davon aus, dass Lernprozesse selbstgesteuerte Konstruktionsprozesse der Schülerinnen und Schüler darstellen, welche die Lehrperson durch Bereitstellung geeigneter Lernumgebungen und anregender Materialien zu initiieren und zu begleiten habe. Die Studierenden wurden zu Bewertungen dieser beiden Sichtweisen, operationalisiert in Form von 14 Items, gebeten.

\section{Transmissionsorientierung}

Die Überzeugung zum Erwerb mathematischen Wissens im Sinne einer Transmissionsorientierung bezieht sich auf eine standardisierte (z.B. „Nicht-standardisierte Verfahren sollten vermieden werden, weil sie das Erlernen des richtigen Verfahrens beeinträchtigen können“), lehrergesteuerte(z.B. ,Schülerinnen und Schüler lernen Mathematik am besten, indem sie den Erklärungen der Lehrperson aufmerksam folgen“) und ergebnisorientierte (z.B. „Man muss ein mathematisches Problem nicht wirklich verstanden haben, Hauptsache man kommt auf die richtige Lösung") Auffassung von mathematischen Wissenserwerbsprozessen (Tabelle 12.4). Lehrkräfte in einem durch das TransmissionsParadigma gekennzeichneten Unterricht fungieren in erster Linie als Wissensvermittelnde, deren Schülerinnen und Schüler vor allem rezeptiv lernen. Die Skala umfasst insgesamt acht Items, die auch hier sechsstufig (,Stimme überhaupt nicht zu“ bis „Stimme völlig zu“) beantwortet wurden. 
Tabelle 12.4: Überzeugung zum Erwerb mathematischen Wissens:

Transmissionsorientierung - Items und Antwortmöglichkeiten

Wie sehr stimmen Sie den folgenden Aussagen über das Lernen von Mathematik zu?

Items

a) Um in Mathematik gut zu sein, muss man sich einfach nur alle Formeln merken.

b) Man muss Schülerinnen und Schülern exakte Verfahren beibringen, damit sie mathematische Probleme lösen können.

c) Man muss ein mathematisches Problem nicht wirklich verstanden haben, Hauptsache man kommt auf die richtige Lösung.

d) Um gut in Mathematik zu sein, muss man Aufgaben schnell lösen können.

e) chülerinnen und Schüler lernen Mathematik am besten, indem sie den Erklärungen der Lehrperson aufmerksam folgen.

f) Wenn Schülerinnen und Schüler sich mit mathematischen Aufgaben befassen, ist das korrekte Ergebnis wichtiger als der Lösungsweg.

g) Nicht-standardisierte Verfahren sollten vermieden werden, weil sie das Erlernen des richtigen Verfahrens beeinträchtigen können.

h) Das Sammeln praxisnaher mathematischer Erfahrungen lohnt sich nicht.

Antwortmöglichkeiten: Stimme überhaupt nicht zu - Stimme nicht zu - Stimme eher nicht zu - Stimme eher zu -Stimme zu -Stimme völlig zu; Cronbach Alpha (CHE). .60 (Prim); .59 (Sek)

Die deskriptiven Analysen ergeben, dass die Deutschschweiz mit Mittelwerten von 8.81 für die Primarstufe bzw. 8.92 für die Sekundarstufe deutlich unter dem neutralen Skalen-Mittelpunkt von 10 liegt und sich im internationalen Ländervergleich jeweils am unteren Ende befindet (Abbildung 12.4). Während die Deutschschweizer Studierenden zur Primarschullehrkraft statistisch signifikant tiefer als ihre Kolleginnen und Kollegen aller anderen Länder liegen, weisen sie auf der Sekundarstufe zusammen mit den USA, Taiwan und Deutschland die tiefsten Werte aus. Die Studierenden sind demnach nicht der Ansicht, dass erfolgreiche mathematische Wissenserwerbsprozesse lehrergesteuert und produktionsorientiert ausgerichtet sein müssen. Auffällig ist, dass die angehenden Lehrpersonen der Deutschschweiz zusammen mit ihren Kolleginnen und Kollegen aus Deutschland diese Position zwar am ausdrücklichsten zum Ausdruck bringen, dass aber auch die Mittelwerte aller anderen Länder unter dem Skalen-Mittelpunkt von 10 und somit im ablehnenden Bereich liegen. Die (noch) höchsten Werte ergeben sich auf der Primar- und Sekundarstufe für Russland (9.65 [Primarstufe] und 9.55 [Sekundarstufe]), Polen (9.50 und 9.19) und Singapur (9.30 und 9.50). Die Streuungen fallen für die Deutschschweiz gering aus ( $\mathrm{SD}=.69$ [Primarstufe]; .77 [Sekundarstufe]), was auf vergleichsweise homogene Überzeugungen hinweist. Die grössten Standardabweichungen zeigen sich für Polen (.82 und .94).

Auch in Betrachtung der Länder-Mittelwerte nach Ausbildungstypen findet sich die Deutschschweiz sowohl auf der Unteren Primarstufe (1-4) mit 
8.72 (Höchstwert Georgien mit 10.19), der Primarstufe (1-6) mit 8.82 (Höchstwert Philippinen mit 10.57) als auch auf der Sekundarstufe I mit 8.92 (Höchstwert Philippinen mit 10.45) an letzter Stelle der Auflistungen. Während auch hier die Unterschiede für die Ausbildungstypen der Primarstufe zu allen anderen Ländern statistisch signifikant ausfallen, zeigen sich für die Sekundarstufe I die Differenzen zu den USA (9.28), Deutschland (8.98) und Norwegen (8.95) im Zufallsbereich, zu allen anderen Ländern jedoch auch statistisch bedeutsam. Wenn auch in dieser Betrachtung die Mittelwerte der einzelnen Länder zumeist im ablehnenden Bereich liegen, so zeigen sich dennoch beachtliche nationale Unterschiede - wie anhand der oben aufgeführten Werte der Deutschschweiz im Vergleich zu den jeweiligen Höchstwerten erkannt werden kann.

Mit Blick auf die Ausbildungsgänge innerhalb der Deutschschweiz erhärtet sich die Vermutung einer vergleichsweise homogen vorliegenden Überzeugung. So befinden sich die Mittelwerte aller Ausbildungsgänge der unterschiedlichen Ausbildungstypen deutlich unter 10 und damit im ablehnenden Bereich (Abbildung 12.4). Trotz dieser Gemeinsamkeit einer ablehnenden Haltung gegenüber dem Erwerb mathematischen Wissens durch Transmission sind die Unterschiede dennoch teilweise statistisch signifikant. So unterscheidet sich auf der Unteren Primarstufe (1-4) U4 von U2 und U1. Auf der Primarstufe (1-6) fallen insbesondere P12 und P13 gegenüber P3, P4, P16 und P5 ab. Und auf der Sekundarstufe I zeigt sich eine Differenz zwischen S4 und S8. Erneut ist erkennbar, dass die Streuungen bei höheren Mittelwerten geringer als bei tieferen rangieren. 
Abbildung 12.4: Überzeugung zum Erwerb mathematischen Wissens:

Transmissionsorientierung - internationaler Ländervergleich und Institutionen der Deutschschweiz

Überzeugung zum Erwerb mathematischen

M SE SD Wissens: Transmissionsorientierung

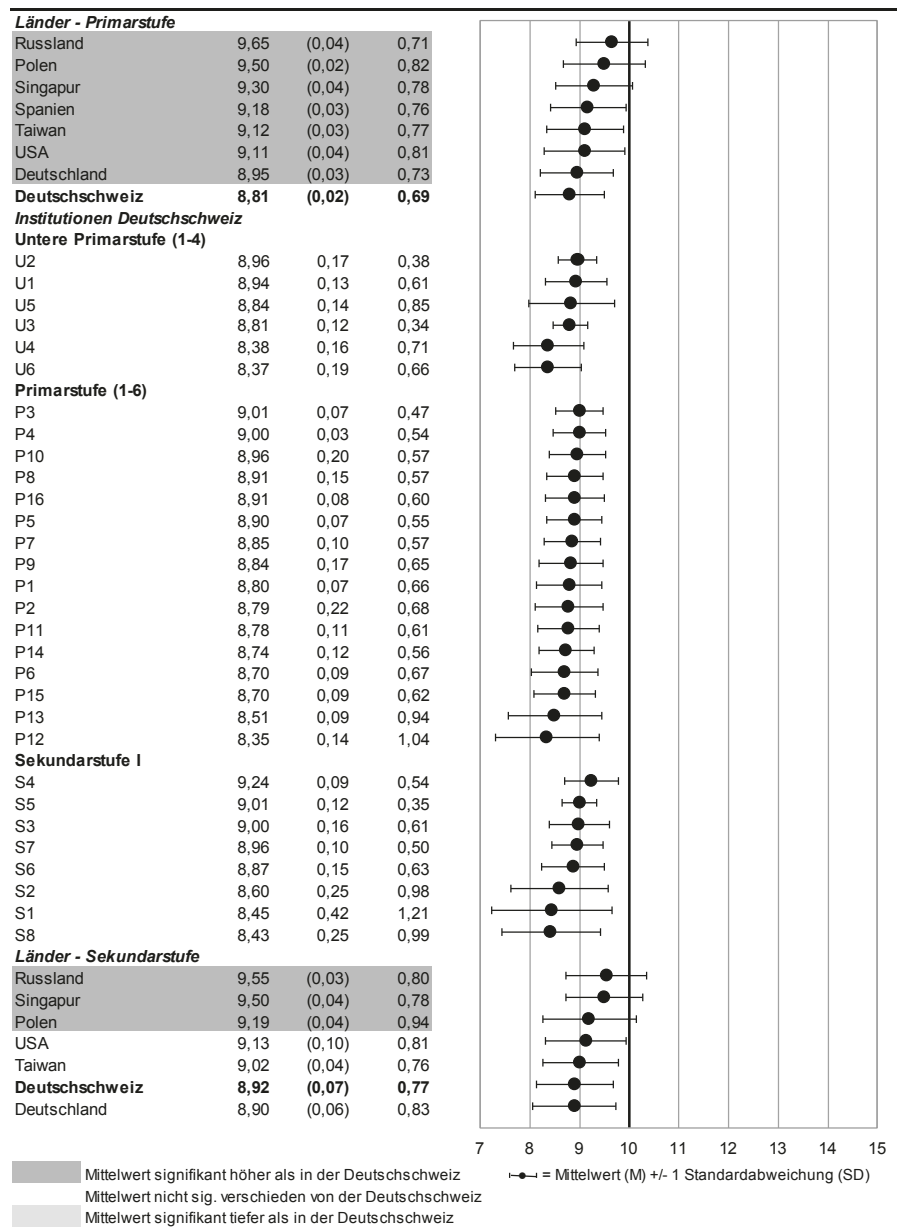

Anmerkung: $\mathrm{M}=$ Mittelwert, $\mathrm{SE}=$ Standardfehler, $\mathrm{SD}=$ Standardabweichung;

Sekundarstufe I =Ausbildungsprogramme mit Unterrichtsberechtigung bis 9. Klassenstufe, Sekundarstufe $=$ Ausbildungsprogramme mit Unterrichtsberechtigung auf Sekundarstufe I und II, d.h. bis Klassenstufe 11/12 (vgl. Kapitel 2 in diesem Band); Abbildung = Mittelwert +/- 1 SD, der theoretische Skalen-Mittelpunkt liegt bei 10, d.h. ein Wert von grösser 10 bedeutet eine Zustimmung und ein Wert von kleiner 10 eine Ablehnung bezüglich des gemessenen Konstrukts. 


\section{Konstruktionsorientierung}

Die Konstruktionsorientierung fokussiert den Erwerb mathematischen Wissens als einen aktiven Konstruktionsprozess durch den Lernenden selbst. Im Mittelpunkt stehen verständnisorientierte und kognitiv aktivierende Lernprozesse, die zu eigenständigen und unterschiedlichen Lösungsfindungen führen (z.B.: „Lehrpersonen sollten Schülerinnen und Schülern die Möglichkeit geben, ihre eigenen Wege zu finden, um eine Aufgabe zu lösen“) (Tabelle 12.5). Nicht die Ergebnisorientierung steht im Vordergrund, sondern der Prozess des mathematischen Lernens. Darüber hinaus wird die Bedeutung des sozialen Austauschs über mathematische Probleme für den Wissenserwerbsprozess betont (z.B.: „Es hilft den Schülerinnen und Schülern, wenn für eine bestimmte Aufgabe verschiedene Lösungswege diskutiert werden"). Die Skala umfasst sechs Items, die sechsstufig („Stimme überhaupt nicht zu“ bis Stimme völlig $\left.\mathrm{zu}^{\prime \prime}\right)$ beantwortet wurden.

Tabelle 12.5: Überzeugung zum Erwerb mathematischen Wissens:

Konstruktionsorientierung - Items und Antwortmöglichkeiten

Wie sehr stimmen Sie den folgenden Aussagen über das Lernen von Mathematik zu?

Items

a) In der Mathematik ist es nicht nur wichtig, die richtige Lösung zu finden, sondern auch zu verstehen, warum diese Lösung richtig ist.

b) Lehrpersonen sollten Schülerinnen und Schülern die Möglichkeit geben, ihre eigenen Wege zu finden, um eine Aufgabe zu lösen.

c) Die Zeit, die man verwendet, um herauszufinden, warum ein Lösungsweg einer mathematischen Aufgabe funktioniert hat, ist sinnvoll genutzte Zeit.

d) Schülerinnen und Schüler können durchaus auch ohne Hilfe der Lehrperson Lösungswege für mathematische Aufgaben finden.

e) Lehrpersonen sollten Schülerinnen und Schüler ermutigen, eigene Lösungen für mathematische Aufgaben zu finden, auch wenn diese nicht effizient sind.

f) Es hilft den Schülerinnen und Schülern, wenn für eine bestimmte Aufgabe verschiedene Lösungswege diskutiert werden.

Antwortmöglichkeiten: Stimme überhaupt nicht zu - Stimme nicht zu - Stimme eher nicht zu - Stimme eher zu - Stimme zu - Stimme völlig zu; Cronbach Alpha (CHE): .64 (Prim); .70 (Sek)

Im internationalen Vergleich liegt die Deutschschweiz sowohl auf der Primarals auch auf der Sekundarstufe an erster Stelle (Abbildung 12.5). Diese „Spitzenpositionen" nimmt sie auf der Primarstufe mit einem Mittelwert von 12.39 zusammen mit Deutschland (12.24) und auf der Sekundarstufe mit einem Mittelwert von 12.49 mit Taiwan (12.36), Deutschland (12.32), USA (12.19) und Polen (12.15) ein. Alle anderen Länder weisen statistisch bedeutsam tiefere Mittelwerte auf, wobei auf beiden Zielstufen Singapur (11.75 [Primarstufe] und 11.54 [Sekundarstufe]) den unteren Abschluss der Rangliste bildet. Ungeachtet dieser Unterschiede liegen die Mittelwerte aller Länder deutlich über 
dem Skalen-Mittelpunkt von 10, so dass die Konstruktionsorientierung also in allen Ländern deutlich Zustimmung erfährt. Studierende sind demzufolge davon überzeugt (und dies insbesondere auch in der Deutschschweiz), dass der Erwerb mathematischen Wissens ein aktiver, selbstständiger Konstruktionsprozess ist, in dessen Mittelpunkt verständnisorientierte und kognitiv aktivierende Lernprozesse stehen. Neben dieser deutlichen Überzeugungszustimmung fällt aber auch auf, dass die Streuungen bzw. Standardabweichungen in allen Ländern beachtlich sind, dies gerade auch im Vergleich mit den Ergebnissen zur Transmissionsorientierung. Während die Studierenden also die (negative) Bewertung einer transmissionsorientierten Perspektive des Lehrens und Lernens sehr ähnlich vornehmen, fallen deren (positiven) Einschätzungen zur konstruktionsorientierten Perspektive doch deutlich heterogener aus.

Betrachtet man die Länderergebnisse nach Ausbildungstypen spiegelt sich das internationale Gesamtergebnis wider. Sowohl auf der Unteren Primarstufe (1-4) mit einem Mittelwert von 12.59 als auch auf der Primarstufe (1-6) mit 12.36 findet sich die Deutschschweiz mit statistisch bedeutsamer Differenz zu allen anderen Ländern an erster Stelle. Am unteren Ende des Vergleichs sind Georgien (10.81) auf der Unteren Primarstufe (1-4) und Singapur (11.72) auf der Primarstufe (1-6) anzutreffen. Auf der Sekundarstufe I befindet sich die Deutschschweiz mit 12.49 ebenfalls in den ersten Rängen des Vergleichs, hier zusammen mit Chile (12.65), USA (12.26), Deutschland (12.12) und Polen (12.09). Den geringsten Mittelwert erreicht Singapur mit 11.67.

Innerhalb der Deutschschweiz liegen die Mittelwerte aller Ausbildungsgänge durchwegs deutlich über dem Skalen-Mittelpunkt von 10 und somit im zustimmenden Bereich (Abbildung 12.5). Auch auf diesem hoch zustimmenden Niveau offenbaren sich zwar Differenzen zwischen den Studiengängen, diese sind jedoch zumeist nicht als statistisch bedeutsam zu werten. Auf der Unteren Primarstufe (1-4) ist einzig der Unterschied zwischen U4 und U3 signifikant. Auf der Primarstufe (1-6) sind es insbesondere P12 (positiv von P16, P11, P13, P4, P5 und P3) und P3 (negativ von P12, P14, P6 und P15), die sich abheben. Und auf der Sekundarstufe I sind es einzig S6 und S7, die sich bedeutsam unterscheiden. Deutlicher fallen hingegen die Differenzen hinsichtlich der Zustimmung innerhalb der Ausbildungsgänge aus - welche aber alle dennoch im positiven Bereich liegen -, wie anhand der Standardabweichungen erkannt werden kann. 
Abbildung 12.5: Überzeugung zum Erwerb mathematischen Wissens:

Konstruktionsorientierung - internationaler Ländervergleich und Institutionen der Deutschschweiz

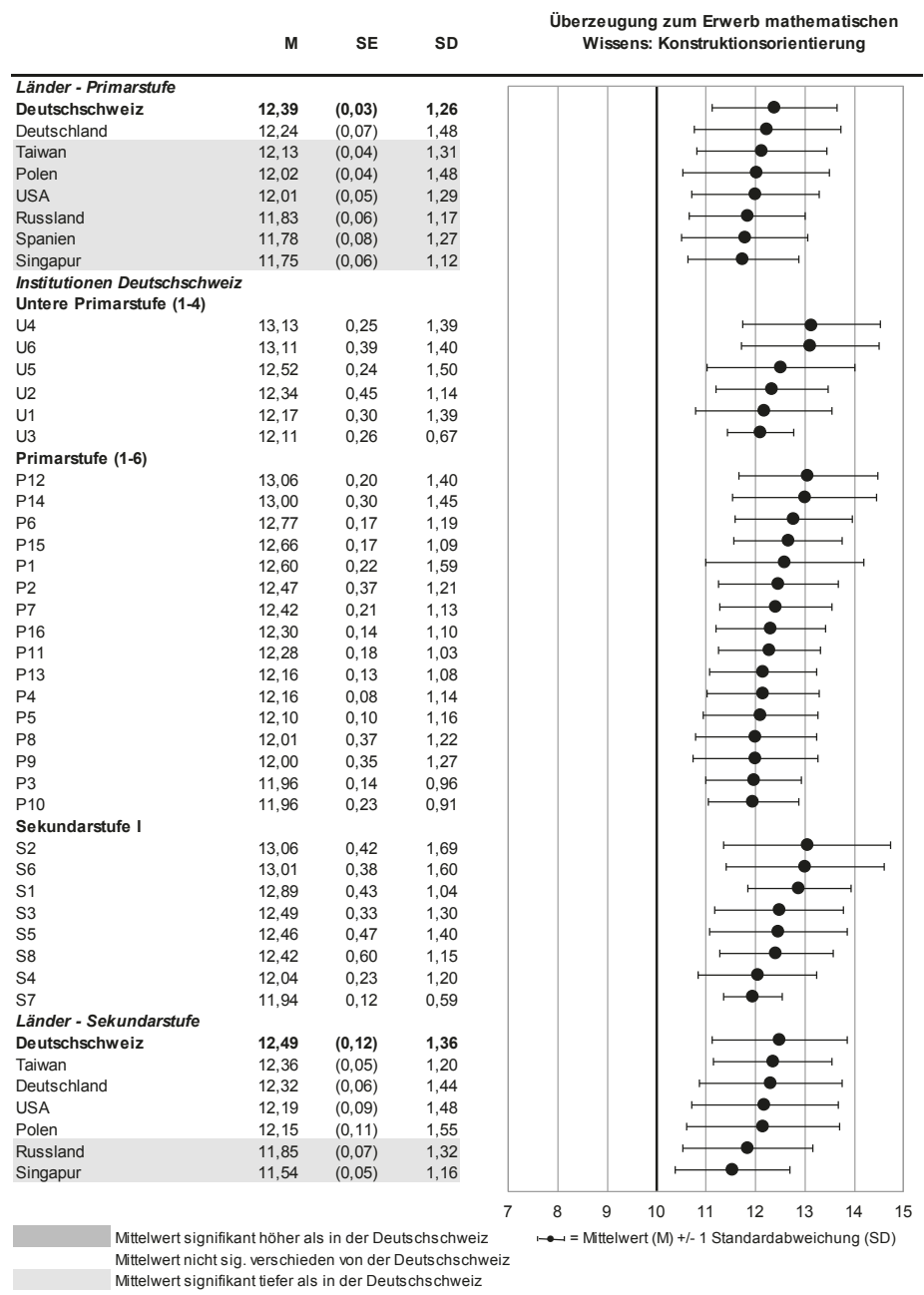

Anmerkung: $\mathrm{M}=$ Mittelwert, $\mathrm{SE}=$ Standardfehler, $\mathrm{SD}=$ Standardabweichung; Sekundarstufe I= Ausbildungsprogramme mit Unterrichtsberechtigung bis 9. Klassenstufe, Sekundarstufe=Ausbildungsprogramme mit Unterrichtsberechtigung auf Sekundarstufe I und II, d.h. bis Klassenstufe 11/12 (vgl. Kapitel 2 in diesem Band); Abbildung = Mittelwert +/- 1 SD, der theoretische SkalenMittelpunkt liegt bei 10, d.h. ein Wert von grösser 10 bedeutet eine Zustimmung und ein Wert von kleiner 10 eine Ablehnung bezüglich des gemessenen Konstrukts. 


\subsection{Zusammenhänge zwischen motivationalen Orientierungen und Überzeugungen zur Mathematik}

Vor dem Hintergrund, dass erst wenig über die Wirkungsweise von berufsbezogenen Überzeugungen von Lehrpersonen auf deren unterrichtliches Handeln bekannt ist (z.B. Müller et al., 2008; Reusser et al., 2011), wird nachfolgend der Frage nachgegangen, welche Zusammenhänge zwischen den Überzeugungen zur Mathematik und den motivationalen Orientierungen angehender Lehrpersonen bestehen. Da die motivationale Orientierung angehender Lehrpersonen im Rahmen der Deutschschweizer Zusatzstudie umfassender erhoben wurde als im internationalen Teil von TEDS-M, basieren die Analysen (nur) auf der Grundlage der Deutschschweizer Stichprobe.

Die motivationale Orientierung (bzw. Berufsmotivation) wurde mittels 16 Items erfasst. Dabei hatten die angehenden Lehrpersonen anzugeben, wie wichtig für sie verschiedene Aussagen für die Wahl des Lehrberufs waren. Die Einschätzungen der Studierenden wurden zu vier Skalen zusammengefasst (für eine umfassende Darstellung vgl. Kapitel 4 in diesem Band): (1) Mit der Skala ,Fachbezogene Motivation “ wurde die Bedeutung des Fachs Mathematik für die Wahl des Lehrberufs erfasst. (2) Bei der ,Extrinsischen Berufsmotivation" geht es um Aspekte wie Lohnattraktivität oder Jobsicherheit. (3) Die Skala „Interesse an Lernenden “ beschreibt die Motivation Lehrperson zu werden, weil jemand gerne mit Kindern oder Jugendlichen arbeitet. (4) Beim „,Interesse an der Vermittlung "steht die pädagogische Einflussnahme als Berufsmotiv im Vordergrund.

Da die Skalen zur motivationalen Orientierung teilweise miteinander korrelieren (vgl. Kapitel 4.3.2 in diesem Band), wurden für die Analysen mehrere multiple Regressionen durchgeführt. Dabei wurden die Skalen der motivationalen Orientierung als Prädiktoren zur Vorhersage der Überzeugung zur Mathematik in die Modelle aufgenommen. Da die Analysen gezeigt haben, dass zwischen den Ausbildungstypen der Unteren Primarstufe (1-4) und der Primarstufe (1-6) keine nennenswerten Unterschiede in den Zusammenhängen zwischen Berufsmotivation und Überzeugungen bestehen, werden im Folgenden die Ergebnisse für beide Ausbildungstypen der Primarstufe gemeinsam dargestellt.

\section{Überzeugungen zur Struktur der Mathematik}

In Abbildung 12.6 ist das Ergebnis zweier multipler Regressionen, eine für die Primarstufe und eine für die Sekundarstufe I, dargestellt. Die Balkenlängen zeigen je die Stärke des Zusammenhangs der Skalen zur motivationalen Orientierung mit einer dynamischen Perspektive zur Struktur der Mathematik. Dabei werden die übrigen Skalen der motivationalen Orientierung konstant gehalten. Statistisch signifikante Zusammenhänge sind dunkel eingefärbt. 
Für die Primarstufe ist der Effekt der fachbezogenen Motivation am stärksten $(B=.43)$. Dies bedeutet, dass bei angehenden Primarlehrpersonen eine um eine Standardabweichung höhere fachbezogene Berufsmotivation mit einem um .43 Punkte höheren Wert in der Skala dynamische Perspektive zur Struktur der Mathematik einhergeht. Ebenfalls signifikant fällt der Zusammenhang zwischen dem Interesse an Lernenden und der dynamischen Perspektive mathematischer Strukturen aus. Auf der Sekundarstufe I ist einzig der Zusammenhang mit der fachbezogenen Motivation statistisch signifikant $(B=.67)$.

Abbildung 12.6: Motivationale Orientierungen und dynamische Perspektive zur Struktur der Mathematik nach Zielstufen in der Deutschschweiz

\section{Dynamische Perspektive}

\section{Primarstufe \\ Fachbezogene Motivation Berufsmotivation extrinsisch Interesse an Lernenden Interesse an Vermittlung \\ Sekundarstufe I \\ Fachbezogene Motivation Berufsmotivation extrinsisch Interesse an Lernenden Interesse an Vermittlung}

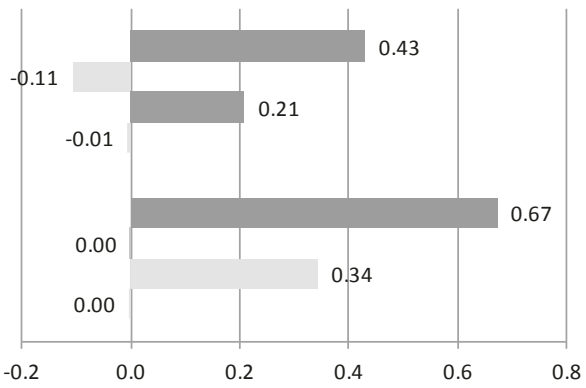

Zusammenhang nicht signifikant Zusammenhang signifikant

Anmerkung: Die Analysen erfolgten mittels multipler linearer Regression. Die Prädiktoren sind für die Deutschschweiz z-standardisiert. Die Balkenlängen entsprechen den unstandardisierten Regressionskoeffizienten (B).

Die Zusammenhänge der Berufsmotivation mit einer statischen Sichtweise zur Struktur der Mathematik sind insgesamt deutlich schwächer ausgeprägt als mit einer dynamischen Perspektive (Abbildung 12.7). Sowohl bei den angehenden Primar- als auch den Sekundarlehrpersonen geht das Interesse an der Vermittlung mit etwas höheren Werten in der statischen Perspektive einher. Zudem hängt die statische Überzeugung bei angehenden Primarlehrpersonen gering $(B=.13)$, aber statistisch signifikant mit einer extrinsischen Berufsmotivation zusammen. Die fachbezogene Motivation kohäriert dagegen schwach negativ mit der statischen Perspektive bezüglich mathematischer Struktur.

Zusammenfassend lässt sich festhalten, dass künftige Lehrpersonen, die eine hohe fachbezogene Motivation als Berufswahlgrund angeben, über ein dynamischeres Verständnis von Mathematik verfügen. Wer den Fachbezug (Mathematik) als weniger wichtig für die Wahl des Lehrberufs erachtet, nimmt eine deutlich statischere Perspektive ein. 
Abbildung 12.7: Motivationale Orientierungen und statische Perspektive zur

Struktur der Mathematik nach Zielstufen in der Deutschschweiz

Statische Perspektive

Primarstufe
Fachbezogene Motivation
Berufsmotivation extrinsisch
Interesse an Lernenden
Interesse an Vermittlung

Sekundarstufe I
Fachbezogene Motivation
Berufsmotivation extrinsisch
Interesse an Lernenden
Interesse an Vermittlung

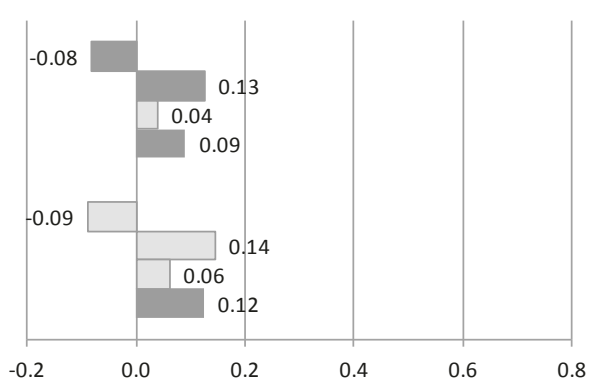

Zusammenhang nicht signifikant

Zusammenhang signifikant

Anmerkung: Die Analysen erfolgten mittels multipler linearer Regression. Die Prädiktoren sind für die Deutschschweiz z-standardisiert. Die Balkenlängen entsprechen den unstandardisierten Regressionskoeffizienten (B).

\section{Überzeugungen zum Erwerb mathematischen Wissens}

Die Zusammenhänge zwischen der transmissionsorientierten Perspektive und den motivationalen Orientierungen fallen insgesamt schwach aus (Abbildung 12.8). Transmissionsorientierung geht einzig bei den angehenden Primarlehrpersonen mit höheren Werten der extrinsischen Berufsmotivation einher.

Bei der Konstruktionsorientierung bestehen engere Zusammenhänge mit der motivationalen Orientierung (Abbildung 12.9). Höhere Transmissionsorientierung ist für die künftigen Primarstufen- und Sekundarstufenlehrpersonen mit einem stärkeren Interesse an den Schülerinnen und Schülern verbunden, wobei der Zusammenhang auf der Sekundarstufe I besonders stark ist $(B=.74)$. Nur auf der Sekundarstufe I zeigt sich ein deutlicher Effekt der fachbezogenen Motivation $(\mathrm{B}=.52)$. Die extrinsische Motivation hat keinen systematischen Zusammenhang mit einer konstruktionsorientierten Überzeugung zum Erwerb mathematischen Wissens. Beim Interesse an der Vermittlung besteht nur bei den Primarlehrpersonen ein geringer Zusammenhang $(B=.16)$ mit der Konstruktionsorientierung.

Insgesamt zeigt sich also, dass eine hohe fachbezogene Motivation der Studierenden nicht nur mit einer dynamischen Perspektive zur Struktur von Mathematik, sondern auch mit einer höheren Konstruktionsorientierung einhergeht. Konstruktionsorientierte Überzeugungen hängen überdies deutlich mit einem hohen Interesse an den Lernenden zusammen. 
Abbildung 12.8: Motivationale Orientierungen und transmissionsorientierte Perspektive zum Erwerb mathematischen Wissens nach Zielstufen in der Deutschschweiz

\section{Transmissionsorientierte Perspektive}

\section{Primarstufe}

Fachbezogene Motivation

Berufsmotivation extrinsisch

Interesse an Lernenden

Interesse an Vermittlung

Sekundarstufe I

Fachbezogene Motivation

Berufsmotivation extrinsisch

Interesse an Lernenden

Interesse an Vermittlung

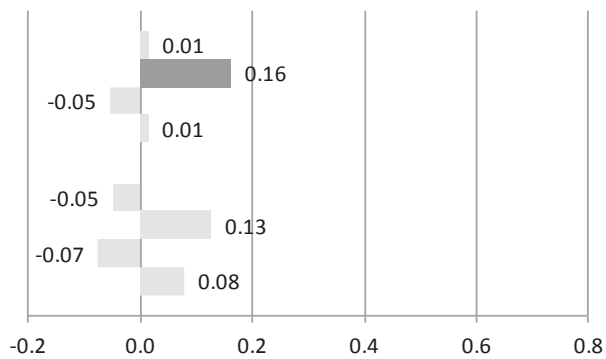

Zusammenhang nicht signifikant

Zusammenhang signifikant

Anmerkung: Die Analysen erfolgten mittels multipler linearer Regression. Die Prädiktoren sind für die Deutschschweiz z-standardisiert. Die Balkenlängen entsprechen den unstandardisierten Regressionskoeffizienten (B).

Abbildung 12.9: Motivationale Orientierungen und konstruktionsorientierte

Perspektive zum Erwerb mathematischen Wissens nach Zielstufen in der Deutschschweiz

\section{Konstruktionsorientierte Perspektive}

\section{Primarstufe}

Fachbezogene Motivation

Berufsmotivation extrinsisch

Interesse an Lernenden

Interesse an Vermittlung

Sekundarstufe 1

Fachbezogene Motivation

Berufsmotivation extrinsisch

Interesse an Lernenden

Interesse an Vermittlung

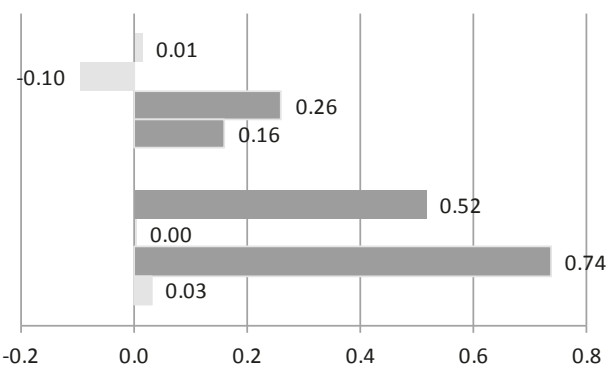

Zusammenhang nicht signifikant

Zusammenhang signifikant

Anmerkung: Die Analysen erfolgten mittels multipler linearer Regression. Die Prädiktoren sind für die Deutschschweiz z-standardisiert. Die Balkenlängen entsprechen den unstandardisierten Regressionskoeffizienten (B). 


\subsection{Fazit: dynamisch-konstruktivistisch-offene Überzeugungs-Syndrome in der Deutschschweiz}

In diesem Beitrag interessierte die Fragestellung nach den Überzeugungen von Studierenden zur Mathematik und zum Erwerb mathematischen Wissens. Neben der Differenzierung in eine statische bzw. dynamische Auffassung über die Struktur der Mathematik wurde erfasst, inwiefern die Überzeugung besteht, dass es sich bei Mathematik um eine angeborene Fähigkeit handelt. Darüber hinaus wurden die Ansichten über mathematische Wissenserwerbsprozesse untersucht. Die interessierende Frage bezog sich hierbei auf die Unterscheidung in eine Transmissionsorientierung und eine Konstruktionsorientierung: Werden eher lehrergesteuerte und produktionsorientierte Wissenserwerbsprozesse in der Mathematik für bedeutsam erachtet oder wird das Lernen im Fach Mathematik eher als ein aktiver Konstruktionsprozess durch den Lernenden selbst verstanden, in dem verständnisorientierte und kognitiv aktivierende Lernprozesse dominieren. Schliesslich interessierte, inwieweit die betrachteten Überzeugungen zur Mathematik mit motivationalen Berufsorientierungen in Zusammenhang stehen.

Die Ergebnisse der vorliegenden Analysen verdeutlichen, dass die epistemologischen Überzeugungen angehender Lehrkräfte in der Deutschschweiz durch ein stärker dynamisches als statisches Verständnis von der Struktur der Mathematik gekennzeichnet sind, gleichwohl sie auch diesbezüglich im Ländervergleich nur mittlere Werte erzielen. Sie sind weiterhin nicht der Auffassung, dass mathematische Fähigkeiten angeboren sind; hier erzielt die Deutschschweiz im Ländervergleich zusammen mit den USA (und Deutschland auf der Sekundarstufe) die niedrigsten bzw. am stärksten ablehnenden Mittelwerte. Korrespondierend hierzu besteht bei Studierenden in der Deutschschweiz die Überzeugung von mathematischen Wissenserwerbsprozessen im Sinne einer Konstruktionsorientierung. Hier erzielt die Deutschschweiz im Ländervergleich bemerkenswerterweise die höchsten Mittelwerte sowohl für die Primarstufe als auch die Sekundarstufe. Demgegenüber zeigen sich für die transmissionsorientierte Sichtweise die klarsten Ablehnungen für die Deutschschweiz.

Die Befunde decken sich zum einen mit der schul- und unterrichtsbezogenen Diskussion im Übergang zum 21. Jahrhundert, in deren Rahmen konstruktionsorientierte Lehr-Lernmethoden bzw. eine stärkere Schülerorientierung als wirksam(er) für Lernentwicklungen gegenüber lehrpersonenzentrierten Unterrichtsarrangements herausgearbeitet wurden (z.B. Hattie, 2009, 2012; Helmke, 2011; Reusser, Pauli \& Waldis, 2010). Wie einleitend bereits vermutet wurde, scheint sich die Entwicklung und Umsetzung derartiger Lehr-Lernmethoden in Schule und Lehrerbildung tatsächlich auch auf die mathematikbezogenen Überzeugungen angehender Lehrkräfte in der Deutschschweiz auszuwirken. Passend zu diesem Ergebnis zeigen sich auch (zumindest tendenziell) Bezie- 
hungen zwischen intrinsischer Berufsmotivation (fachbezogene Motivation, Interesse an Lernenden) und dynamisch-konstruktivistischen Überzeugungen zur Mathematik. Konkret zeigt sich, dass Studierende, die über eine dynamische Überzeugung hinsichtlich der Struktur der Mathematik verfügen, eine höhere fachbezogene Motivation signalisieren. Darüber hinaus sind dynamische Überzeugungen bei angehenden Primarstufenlehrkräften mit einem stärkeren Interesse an den Lernenden verbunden. Ebenfalls kohäriert das Interesse an der Vermittlung positiv mit höheren Werten der statischen Perspektive und dies bei Primar- und Sekundarstufenlehrkräften. Weiterhin sind Studierende der Primarstufe, die eine statische Überzeugung von der Struktur der Mathematik vertreten, eher extrinsisch motiviert, den Lehrerberuf zu ergreifen, und zeigen eine geringere fachbezogene Motivation. Die Analysen zu den $\mathrm{Zu}-$ sammenhängen zwischen der Transmissions- bzw. Konstruktionsorientierung beim mathematischen Wissenserwerb und den motivationalen Orientierungen ergeben weniger eindeutige Befunde. Bei angehenden Primarlehrpersonen konnte einzig ein signifikanter positiver Zusammenhang zwischen der Transmissionsorientierung und der extrinsischen Berufsmotivation nachgewiesen werden. Für die Konstruktionsorientierung liessen sich vier signifikante Effekte belegen: Studierende der Primarstufe, die eine konstruktivistische Überzeugung zum Erwerb mathematischen Wissens besitzen, zeigen ein höheres Interesse an Lernenden und an Vermittlung; Studierende der Sekundarstufe verfügen über eine höhere fachbezogene Motivation sowie ein höheres Interesse an Lernenden.

Die Ergebnisse korrespondieren zudem mit der Annahme, dass Überzeugungen kulturspezifisch geprägt werden. In der kulturvergleichenden Psychologie sind kulturelle Unterschiede anhand verschiedener wertebezogener Dimensionen erfasst und systematisiert worden. Hofstede (2001) etwa unterscheidet fünf Kulturdimensionen, innerhalb derer einzelne Länder aufgrund empirisch gewonnener Werte in eine Rangfolge gebracht werden. Die Dimension „Individualismus vs. Kollektivismus“ verdeutlicht, wie stark ein Land individualistisch bzw. kollektivistisch ausgerichtet ist. Sehr hohe Individualismuswerte erzielen nach dem Hofstede-Index englischsprachige Länder wie etwa die USA, Australien, Großbritannien und Kanada. Asiatische Länder wie Malaysia, Thailand und Indonesien erreichen dagegen deutlich geringere Individualismuswerte. In TEDS-M 2008 wurde der Individualismus-Kollektivismus-Index nach Hofstede herangezogen, um die kulturellen Prägungen der Teilnehmerländer zu unterscheiden. Es zeigte sich, dass die Überzeugungsstrukturen der einzelnen Länder weitgehend mit der Einordnung dieser Länder auf dem Individualismus-Kollektivismus-Index von Hofstede korrespondieren, wenngleich sich mehr ein Kontinuum als scharf abgrenzbare Gruppen bilden lassen. So vertraten Lehramtsstudierende aus kollektivistisch orientierten Ländern im internationalen Vergleich stärker eine statische Überzeugung zur Struktur der Mathematik und eine transmissionsorientierte Überzeugung zum Erwerb mathematischen Wissens. Die Schweiz kann der Gruppe der indivi- 
dualistisch orientierten Länder zugeordnet werden, und die dargestellten Ergebnisse der Deutschschweiz weisen entsprechend in die erwartete Richtung einer kulturell geprägten Überzeugungsstruktur, die durch dynamische und konstruktionsorientierte Überzeugungen charakterisiert ist. Die Ergebnisse korrespondieren mit Befunden aus anderen Untersuchungen. In der TALISStudie (Teaching and Learning International Survey) konnten 25\% der Varianz konstruktivistischer Überzeugungen und 50\% der Varianz transmissionsorientierter Überzeugungen durch die Länderzugehörigkeit aufgeklärt werden (Blömeke et al., 2010; OECD, 2009; Schmotz, Felbrich \& Kaiser, 2010).

Epistemologische Überzeugungen gelten als bedeutsam für das Unterrichtshandeln von Lehrkräften, indem die Wahrnehmung von Unterrichtsprozessen vorstrukturiert und die Verarbeitung der wahrgenommenen Informationen beeinflusst wird (u.a. Calderhead, 1996; Leder \& Forgasz, 2002). In der weiteren Wirkungskette wird angenommen, dass epistemologische Überzeugungen über das Unterrichtshandeln einen Einfluss auf Lernprozesse von Schülerinnen und Schülern ausüben. Die empirische Evidenz für den angenommenen Einfluss dieser Vorstellungen und subjektiven Theorien über Wissen und Wissenserwerb ist gleichwohl noch gering. Epistemologische Überzeugungen wurden bislang häufig bei Schülerinnen und Schülern und hierbei domänenspezifisch im Fach Mathematik bzw. in den Naturwissenschaften untersucht. Studien, die den Zusammenhang zwischen epistemologischen Überzeugungen von Lehrkräften und ihrem Unterrichtshandeln sowie Wirkungen auf Leistungen der Schülerinnen und Schüler in den Blick nehmen, liegen dagegen wenige vor. Peterson, Fennema, Carpenter und Loef (1989) ermittelten einen Zusammenhang zwischen den Überzeugungen von Primarstufenlehrkräften und ihrer Unterrichtsgestaltung. Lehrpersonen, die eine konstruktivistische Überzeugung über das Lernen von Mathematik besassen, verwendeten mehr Zeit für kognitiv anregende Problemlöseaufgaben und weniger Zeit für die Vermittlung von Faktenwissen im Vergleich zu Lehrpersonen, die eine transmissionsorientierte Auffassung über Lernen von Mathematik vertraten. Staub und Stern (2002) konnten ebenso eine handlungsleitende Funktion von epistemologischen Überzeugungen belegen. Lehrpersonen mit konstruktivistischen Überzeugungen setzten in ihrem Unterricht mehr Aufgaben ein, die ein anspruchsvolleres konzeptuelles Mathematikverständnis erfordern. Die Forschenden konnten zudem einen positiven Effekt konstruktivistischer Überzeugungen auf die Leistungsentwicklung der Schülerinnen und Schüler nachweisen. In einer Untersuchung von Stipek, Givvin, Salmon und MacGyvers (2001) zeigten sich ebenfalls bedeutsame Zusammenhänge zwischen den epistemologischen Überzeugungen über Mathematik und der Unterrichtsgestaltung. Grundschullehrkräfte mit stärker statischen Überzeugungen gestalteten ihren Unterricht u.a. weniger verständnisorientiert und autonomiefördernd und stärker ergebnisorientiert. Nachteilige Effekte einer Transmissionsorientierung in den Überzeugungen von Lehrkräften im Hinblick auf eine weniger kognitiv aktivierende Unterrichtsgestaltung konnten darüber hinaus anhand der 
COACTIV-Daten belegt werden (Dubberke, Kunter, McElvany, Brunner \& Baumert, 2008).

Wenngleich durch verschiedene Studien eine handlungsleitende Wirkung epistemologischer Überzeugungen und ein Zusammenhang zur Unterrichtsgestaltung belegt ist, muss beachtet werden, dass die Studien zumeist kleine Stichproben verwendeten und häufig auf Selbstberichten der Lehrkräfte zu ihrer Unterrichtspraxis basieren. Zudem ist die Befundlage nicht eindeutig. Es liegen auch Untersuchungen vor, die keine Zusammenhänge zwischen epistemologischen Überzeugungen und dem Unterrichtshandeln und Unterrichtswirkungen nachweisen konnten (u.a. Galton, Simon \& Croll, 1980; Seidel, Schwindt, Rimmele \& Prenzel, 2008). Die Untersuchung von Seidel, Schwindt, Rimmele und Prenzel (2008), die Daten der IPN-Videostudie nutzte, konnte auf der Ebene von beobachtetem Unterricht keine signifikanten Zusammenhänge zwischen den konstruktivistischen Überzeugungen von Lehrkräften und dem Wissenszuwachs bzw. der Interessenentwicklung bei Schülerinnen und Schülern nachweisen.

Hinsichtlich der Lehrerausbildung stellt sich die Frage nach möglichen Modifikationen von epistemologischen Überzeugungen. Im Allgemeinen gelten epistemologische Überzeugungen als relativ stabile kognitive Strukturen, die wenig veränderungssensitiv sind. Im Wahrnehmungsprozess übernehmen sie die Funktion der Filterung von Informationen und strukturieren diese vor. Die Verarbeitung von Ausbildungsinhalten wird also von Studienbeginn an beeinflusst und gefiltert. Analog zu subjektiven Theorien sind epistemologische Überzeugungen in der Regel unbewusst und unreflektiert, können aber unter bestimmten Bedingungen expliziert werden (Groeben, Wahl, Schlee \& Scheele, 1988).

Aus Sicht der Lehrerausbildung erscheint es zielführend, bei Studierenden den Aufbau jener epistemologischen Überzeugungen zu unterstützen, die positiv mit Unterrichtsqualität und Leistungen der Schülerinnen und Schüler assoziiert sind, und diejenigen naiven Theorien zu reduzieren, die in einem negativen Zusammenhang zur Qualität von Unterricht stehen. Angesichts der oben erwähnten empirischen Befundlage, die tendenziell darauf hindeutet, dass sich konstruktivistische Überzeugungen positiv auf die Unterrichtsqualität und die Leistungen der Schülerinnen und Schüler auswirken, ist eine diesbezügliche Förderung anzustreben (Voss, Kleickmann, Kunter \& Hachfeld, 2011).

Veränderungsprozesse von Überzeugungen sind u.a. vor dem Hintergrund von Modellen des „Conceptual Change“ diskutiert worden, die auch in der Domäne der Mathematik Anwendung fanden und die sich nach Patrick und Pintrich (2001) in Lehr-Lernkontexten als erfolgreich erwiesen haben. Wie die Autoren resümieren, ist die Modifikation epistemologischer Überzeugungen gleichwohl ein zeitaufwändiger, schwieriger und langfristiger Prozess, der eine intensive Auseinandersetzung mit den Überzeugungen und nicht unerhebliche (meta-)kognitive Anstrengungen erforderlich machen, die im Kontext der Lehrerausbildung oftmals auch unter dem Begriff der Selbstreflexion gefasst 
werden. Selbstreflexive Prozesse werden relativ übereinstimmend als eine entscheidende Voraussetzung bewertet, um die eigenen naiven Theorien zu verändern (Reusser et al., 2011). Das Ausmass der Conceptual-Change-Prozesse ist weiterhin abhängig von den Überzeugungen, die Lehramtsstudierende im Verlauf ihrer biografischen Entwicklung ausgebildet haben und die bestimmend für die Wahrnehmung und Verarbeitung von Lerninhalten in der Ausbildung sind. So können auch veränderungsresistente Überzeugungen vorliegen, oder es wurden auch unvollständige Veränderungsprozesse beobachtet (Patrick \& Pintrich, 2001). Es gibt darüber hinaus Hinweise für eine Koexistenz unterschiedlicher Überzeugungen, indem im Verlauf der Ausbildung nicht eine Dimension von Überzeugungen durch eine andere ersetzt wird, sondern beide fortbestehen. Daher erscheint es relevant, bei (Um-)Strukturierungsprozessen von epistemologischen Überzeugungen jeweils beide Dimensionen zu berücksichtigen und zu bearbeiten. Bemühungen, Überzeugungen von (angehenden) Lehrkräften reflexiv zugänglich zu machen und somit zu verändern, sind darüber hinaus offenbar wirkungsvoller, wenn sie innerhalb der Ausbildung bzw. in der Berufseingangsphase stattfinden und nicht erst in der (späteren) Phase der Berufstätigkeit. So konnte ebenfalls basierend auf Daten von TEDS-M jüngst aufgezeigt werden, dass mathematikdidaktische Lerngelegenheiten im Rahmen der Lehrerausbildung in bedeutsamer Beziehung mit dynamisch-konstruktivistischen Überzeugungen bei Lehramtsstudierenden stehen (Biedermann, Brühwiler \& Krattenmacher, 2012; Biedermann, Brühwiler \& Steinmann, 2012). Zudem gibt es Hinweise dafür, dass verschiedene Kontextfaktoren und Lehrermerkmale eine Veränderung behindern (z.B. geringes Fachwissen) oder begünstigen können (Reusser et al., 2011).

Im Hinblick auf praktische Folgerungen für die Aus- und Weiterbildung von Lehrpersonen stellen Patrick und Pintrich (2001) generelle Bedingungen und Zielperspektiven heraus. Demnach ist es bedeutsam, dass (1) Lehrerbildende ein Bewusstsein für die Überzeugungen ihrer Studierenden haben, (2) die Lehrerbildung hinreichend Lerngelegenheiten bereithält, um Studierenden ein Bewusstwerden und eine Auseinandersetzung mit den eigenen Überzeugungen zu ermöglichen, (3) die Lernumgebung hinreichend unterstützend gestaltet ist, um Conceptual-Change-Prozesse zu fördern und (4) die verschiedenen Überzeugungen der Studierenden explizit gemacht und diskutiert werden. Konkrete Konzepte zur Veränderung der epistemologischen Überzeugungen von Lehramtsstudierenden, die auf Conceptual-Change-Ansätzen basieren, sind insbesondere im angloamerikanischen Sprachraum ausgearbeitet und empirisch untersucht worden. So entwickelten Feiman-Nemser, McDiarmid, Melnick und Parker (1989) an der Michigan State Universität einen Workshop, in dem sich Studierende u.a. durch Analyse von Fallstudien, videografierten Unterricht, Diskussionen und Simulationen mit verschiedenen Themen über Unterricht auseinandersetzen. In einer Variante des Kurses verbringen die Studierenden vier Tage an einer Schule und reflektieren Unterricht unter jeweils ausgewählten Beobachtungsschwerpunkten. Gill, Ashton und 
Algina (2004) konnten unter Einbezug einer Kontrollgruppe positive Effekte einer speziellen Intervention zur Bearbeitung mathematischer Überzeugungen belegen. Auch Kleickmann, Möller und Jonen (2006) wiesen nach, dass durch Fortbildungen im Bereich des naturwissenschaftlichen Lehrens und Lernens in der Grundschule, die auf Conceptual-Change-Ansätzen basieren, konstruktivistische Überzeugungen bei Lehrkräften gefördert werden konnten (Voss et al., 2011).

Alles in allem existiert inzwischen eine Reihe an Studien, die sich mit der Frage beschäftigen, wie sich Überzeugungen durch spezifische Kurse modifizieren lassen. Dabei konnte allerdings auch gezeigt werden, dass nicht intendierte Wirkungen eintreten können und dass sich Veränderungen von Überzeugungen durch entsprechende Programme z.T. nicht auf das tatsächliche Unterrichtshandeln auswirken (Reusser et al., 2011). Für die Konzeption von Programmen sind die Anregung (selbst-)reflexiver Lernprozesse und die Vermittlung neuen Wissens zwar zentrale, aber nicht hinreichende Gelingensbedingungen - wie auch Reusser et al. (2011) in ihrem Überblickartikel herausstellen. Als ebenso wichtig wird die Einbettung in situierte, problem- und handlungsorientierte - inklusive videobasierte - Lernkontexte erachtet, in denen Inputphasen mit unterrichtsbezogenen Trainings-, Reflexions- und Feedbackphasen kombiniert werden. Neben dem fachspezifisch-pädagogischen Coaching (Staub, 2001) verweisen die Autorinnen und Autoren auf verschiedene Mentoring- und Trainingsprogramme unter anderem im Forschungskontext der Subjektiven Theorien, die sich als erfolgreich erwiesen haben. Die Möglichkeiten und Bedingungen einer systematischen Implementation derartiger Programme in die Lehrerausbildung erfordern weiterführende Forschungsbemühungen, um auch die Wirksamkeit etwa im Hinblick auf das tatsächliche Handeln der Lehrpersonen oder hinsichtlich der Unterrichtswirkungen zu belegen.

Abschliessend kann festgehalten werden, dass die Ergebnisse somit die einleitend aufgestellten Vermutungen insgesamt bestätigen. Es zeigen sich die angenommenen dynamisch-konstruktivistisch-offenen ÜberzeugungsSyndrome hinsichtlich der Struktur und des Lehrens und Lernens von Mathematik. Teilweise erwartungswidrig offenbaren sich einzig die Ergebnisse für die Ausbildungsinstitutionen der Deutschschweiz. Während die Ergebnisse zur statischen Struktur und transmissionsorientierten Sichtweise noch relativ homogen tief und somit erwartungskonform ausfallen, zeigen sich bezüglich der dynamischen Struktur und konstruktionsorientierten Sichtweise teilweise grosse Differenzen. Worauf diese Unterschiede gründen, kann anhand der vorliegenden Daten nicht erklärt werden - hier wäre es interessant, in einer Anschlussstudie vertiefende Analysen durchzuführen. Auch lässt sich nicht abschliessend bewerten, welche diesbezüglichen Ergebnisse für die zukünftige Lehrtätigkeit als vorteilhaft zu betrachten sind. Einerseits wurde bereits mehrmals erwähnt, dass sich in den letzten Jahren die Erkenntnis durchgesetzt hat, dass konstruktivistische Lehr-Lernarrangements eher positive Lernentwick- 
lungen bei Schülerinnen und Schülern erwarten lassen. Andererseits zeigt sich diese Forschungslage keineswegs positiv und wird auch immer wieder auf die adaptive Kompetenz erfolgreicher Lehrpersonen verwiesen (Beck et al., 2008; Brühwiler, 2014). Dabei kann zumindest vermutet werden, dass sich Adaptivität auch in der situationsangepassten Anwendung konstruktions- und transmissionsorientierter Lehr-Lernarrangements zeigt. Auch dieser Vermutung würde es sich lohnen, in einer weiteren Forschungsarbeit nachzugehen.

\subsection{Literatur}

Baumert, J. \& Kunter, M. (2006). Stichwort: Professionelle Kompetenz von Lehrkräften. Zeitschrift für Erziehungswissenschaften, 9(4), 469-520.

Baumert, J., Kunter, M., Brunner, M., Krauss, S., Blum, W. \& Neubrand, M. (2004). Mathematikunterricht aus Sicht der PISA-Schülerinnen und -Schüler und ihrer Lehrkräfte. In M. Prenzel, J. Baumert, W. Blum, R. Lehmann, D. Leutner, M. Neubrand et al. (Hrsg.), PISA 2003. Der Bildungsstand der Jugendlichen in Deutschland - Ergebnisse des zweiten internationalen Vergleichs. Münster: Waxmann, S. 314-354.

Beck, E., Baer, M., Guldimann, T., Bischoff, S., Brühwiler, C., Müller, P. et al. (2008). Adaptive Lehrkompetenz: Analyse und Struktur, Veränderbarkeit und Wirkung handlungssteuernden Lehrerwissens. Münster: Waxmann.

Biedermann, H., Brühwiler, C. \& Krattenmacher, S. (2012). Lernangebote in der Lehrerausbildung und Überzeugungen zum Lehren und Lernen. Zeitschrift für Pädagogik, 58(4), 460-475.

Biedermann, H., Brühwiler, C. \& Steinmann, S. (2012). Making the Impossible Possible? Establishing Beliefs about Teaching and Learning during Teacher Training Courses. In J. König (Hrsg.), Teachers' Pedagogical Beliefs: Definition and Operationalisation - Connections to Knowledge and Performance-Development and Change (S. 37-52). Münster: Waxmann.

Blömeke, S., Müller, C., Felbrich, A. \& Kaiser, G. (2008). Epistemologische Überzeugungen zur Mathematik. In S. Blömeke, G. Kaiser \& R. Lehmann (Hrsg.), Professionelle Kompetenz angehender Lehrerinnen und Lehrer: Wissen, Überzeugungen und Lerngelegenheiten deutscher Mathematikstudierender und-referendare. Erste Ergebnisse zur Wirksamkeit der Lehrerausbildung (S. 219-246). Münster: Waxmann.

Blömeke, S., Kaiser, G. \& Lehmann, R. (2010). TEDS-M 2008 Sekundarstufe I: Ziele, Untersuchungsanlage und zentrale Ergebnisse. In S. Blömeke, G. Kaiser \& R. Lehmann (Hrsg.), TEDS-M 2008. Professionelle Kompetenz und Lerngelegenheiten angehender Mathematiklehrkräfte für die Sekundarstufe I im internationalen Vergleich (S. 11-38). Münster: Waxmann. 
Bråten, I., Britt, M. A., Strømsø, H. I. \& Rouet, J. F. (2011). The Role of Epistemic Beliefs in the Comprehension of Multiple Expository Texts: Towards an Integrated Model. Educational Psychologist, 46, 48-70.

Brühwiler, C. (2014). Adaptive Lehrkompetenz und schulisches Lernen. Münster: Waxmann.

Calderhead, J. (1996). Teachers: Beliefs and Knowledge. In D. C. Berliner \& R. C. Calfee (Hrsg.), Handbook of Educational Psychology (S. 709-725). New York: Macmillan.

Dann, H.-D., Müller-Fohrbrodt, G. \& Cloetta, B. (1981). Sozialisation junger Lehrer im Beruf: „Praxisschock“ drei Jahre später. Zeitschrift für Entwicklungspsychologie und Pädagogische Psychologie, 13, 251-262.

Drechsel, B. (2001). Subjektive Lernbegriffe und Interesse am Thema Lernen bei angehenden Lehrpersonen. Münster: Waxmann.

Dubberke, T., Kunter, M., McElvany, N., Brunner, M. \& Baumert, J. (2008). Lerntheoretische Überzeugungen von Mathematiklehrkräften: Einflüsse auf die Unterrichtsgestaltung und den Lernerfolg von Schülerinnen und Schülern. Zeitschrift für Pädagogische Psychologie, 22(3-4), 193-206.

Feiman-Nemser, S., McDiarmid, G. W., Melnick, S. L. \& Parker, M. (1989). Changing Beginning Teachers ' Conceptions: A Description of an Introductory Teacher Education Course. East Lansing, MI: The National Center for Research on Teacher Education.

Fenstermacher, G. D. (1994). The Knower and the Known: The Nature of Knowledge in Research on Teaching. In L. Darling Hammond (Hrsg.), Review of Research in Education (S. 3-56). Washington, DC: American Educational Research Association.

Forgasz, H. \& Leder, G. C. (2008). Beliefs about Mathematics and Mathematics Teaching. In P. Sullivan \& T. Wood (Hrsg.), International Handbook of Mathematics Teacher Education: Vol. 1. Knowledge and Beliefs in Mathematics Teaching and Teaching Development (S. 173-192). Rotterdam: Sense Publishers.

Galton, M., Simon, B. \& Croll, P. (1980). Inside the Primary Classroom. London: Routledge \& Kegan Paul.

Gill, M. G., Ashton, P. T. \& Algina, J. (2004). Changing Preservice Teachers' Epistemological Beliefs about Teaching and Learning in Mathematics: An Intervention Study. Contemporary Educational Psychology, 29(2), 164-185.

Goldin, G., Rösken, B. \& Törner, G. (2009). Beliefs - No Longer a Hidden Variable in Mathematical Teaching and Learning Processes. In J. Maass \& W. Schlöglmann (Hrsg.), Beliefs and Attitudes in Mathematics Education: New Research Results (S. 1-18). Rotterdam: Sense Publishers.

Grigutsch, S., Raatz, U. \& Törner, G. (1998). Einstellungen gegenüber Mathematik bei Mathematiklehrern [Mathematics Teachers' Epistemological Beliefs about the Nature of Mathematics]. Journal für Mathematik-Didaktik, 19, 3-45. 
Groeben, N., Wahl, D., Schlee, J. \& Scheele, B. (1998). Das Forschungsprogramm subjektive Theorien. Tübingen: Francke.

Hattie, J. (2009). Visible Learning. A Syntheses of Over 800 Meta-Analyses Relating to Achievement. London: Routledge.

Hattie, J. (2012). Visible Learning for Teachers. London: Routledge.

Helmke, A. (2011). Forschung zur Lernwirksamkeit des Lehrerhandelns. In E. Terhart, H. Bennewitz \& M. Rothland (Hrsg.), Handbuch der Forschung zum Lehrerberuf (S. 630-643). Münster: Waxmann.

Hofer, B. K. (2001). Personal Epistemology Research: Implications for Learning and Transfer. Educational Psychology Review, 13(4), 353-383.

Hofer, B. K. \& Pintrich, P. R. (Hrsg.) (2002). Personal Epistemology: The Psychology of Beliefs about Knowledge and Knowing. Mahwah, N.J.: Erlbaum.

Hofstede, G. (2001). Culture's Consequences - Comparing Values, Behaviors, Institutions and Organizations Across Nations. London, Neu Delhi: Thousand Oaks.

Kleickmann, T., Möller, K. \& Jonen, A. (2006). Die Wirksamkeit von Fortbildungen und die Bedeutung von tutorieller Unterstützung. In R. Hinz \& T. Pütz (Hrsg.), Professionelles Handeln in der Grundschule: Entwicklungslinien und Forschungsbefunde (S. 121-128). Baltmannsweiler: Schneider Verlag Hohengehren.

Koch, J.-J. (1972). Lehrer-Studium und Beruf. Einstellungswandel in den beiden Phasen der Ausbildung. Ulm: Süddeutsche Verlagsgesellschaft.

König, J. (Hrsg.) (2012). Teachers' Pedagogical Beliefs. Definition and Operationalisation - Connections to Knowledge and Performance - Development and Change. Münster: Waxmann.

Leder, G. C. \& Forgasz, H. J. (2002). Measuring Mathematical Beliefs and Their Impact on the Learning of Mathematics: A New Approach. In G. C. Leder, E. Pehkonen \& G. Toerner (Hrsg.), Beliefs - a Hidden Variable in Mathematics Education? (S. 73-94). Dordrecht: Kluwer.

Lester, F. K. (Hrsg.) (2007). Second Handbook of Research on Mathematics Teaching and Learning. Charlotte, NC: National Council of Teachers of Mathematics \& Information Age Publishing.

Lortie, D. (1975). Schoolteacher. Chicago: University of Chicago Press.

Müller, C., Felbrich, A. \& Blömeke, S. (2008). Überzeugungen zum Lehren und Lernen von Mathematik. In S. Blömeke, G. Kaiser \& R. Lehmann (Hrsg), Professionelle Kompetenz angehender Lehrerinnen und Lehrer S. 247-276). Münster: Waxmann.

Müller-Fohrbrodt, G., Cloetta, B. \& Dann, H. D. (1978). Der Praxisschock bei jungen Lehrern. Stuttgart: Klett Verlag.

Niederhauser, D. S. \& Stoddart, T. (2001). Teachers'Instructional Perspectives and Usw of Educational Software. Teaching and Teacher Education, 17, $15-31$. 
OECD (2009). Creating Effective Teaching and Learning Environments. First Results from TALIS. Paris: OECD-Publishing

Op't Eynde, P., De Corte, E. \& Verschaffel, L. (2002). Framing Students“ Mathematics Related Beliefs. A Quest for Conceptual Clarity and a Comprehensive Categorization. In G. C. Leder, E. Pehkonen \& G. Törner (Hrsg.), Beliefs: A Hidden Variable in Mathematics Education (S. 13-37)? Netherlands: Kluwer Academic Publishers.

Oser, F. \& Blömeke S. (2012). Überzeugungen von Lehrpersonen. Einführung in den Thementeil. Zeitschrift für Pädagogik, 58(4), 415-421.

Pajares, F. (1992). Teachers' Beliefs and Educational Research: Cleaning Up a Messy Construct. Review of Educational Research, 62, 307-332.

Patrick, H. \& Pintrich, P. R. (2001). Conceptual Change in Teachers' Intuitive Conceptions of Learning, Motivation, and Instruction: The Role of Motivational and Epistemological Beliefs. In B. Torff \& R. J. Sternberg (Hrsg.), Understanding and Teaching the Intuitiv Mind: Student and Teaching Learning (S. 117-143). Mahwah, NJ: Erlbaum.

Peterson, P. L., Fennema, E., Carpenter, T. P. \& Loef, M. F. (1989). Teachers“ Pedagogical Content Beliefs in Mathamatics. Cognition and Instruction, $6(1), 1-40$.

Reusser, K., Pauli, C. \& Waldis, M. (Hrsg.) (2010). Unterrichtsgestaltung und Unterrichtsqualität. Ergebnisse einer internationalen und schweizerischen Videostudie zum Mathematikunterricht. Münster: Waxmann.

Reusser, K., Pauli, C. \& Elmer, A. (2011). Berufsbezogene Überzeugungen von Lehrerinnen und Lehrern. In E. Terhart, H. Bennewitz, M Rothland (Hrsg.), Handbuch der Forschung zum Lehrerberuf (S. 478-495). Münster: Waxmann.

Richardson, V. (1996). The Role of Attitudes and Beliefs in Learning to Teach. In J. Sikula (Hrsg.), Handbook of Research on Teacher Education (2nd ed.) (S. 102-119). New York: Macmillan.

Schmotz, C., Felbrich, A. \& Kaiser, G. (2010). Überzeugungen angehender Mathematiklehrkräfte für die Sekundarstufe I im internationalen Vergleich. In S. Blömeke, G. Kaiser \& R. Lehmann (Hrsg.), TEDS-M 2008. Professionelle Kompetenz und Lerngelegenheiten angehender Mathematiklehrkräfte für die Sekundarstufe I im internationalen Vergleich (S. 279-305). Münster: Waxmann.

Schoenfeld, A. H. (1998). Toward a Theory of Teaching-In-Context. Berkeley, CA: University of California

Seidel, T., Schwindt, K., Rimmele, R. \& Prenzel, M. (2008). Konstruktivistische Überzeugungen von Lehrpersonen: Was bedeuten sie für den Unterricht? In M. A. Meinert, M. Prenzel \& S. Hellekamps (Hrsg.), Perspektiven der Didaktik (S. 259-276). Wiesbaden: VS Verlag für Sozialwissenschaften. 
Staub, F. C. (2001). Fachspezifisch-pädagogisches Coaching: Förderung von Unterrichtsexpertise durch Unterrichtsentwicklung. Beiträge zur Lehrerbildung, 19(2), 175-198.

Staub, F. C. \& Stern, E. (2002). The Nature of Teachers' Pedagogical Content Beliefs Matters for Students' Achievement Gains: Quasi-Experimental Evidence From Elementary Mathematics. Journal of Educational Psychology, 94(2), 344-355.

Stipek, D., Givvin, K., Salmon, J. \& MacGyvers, V. (2001). Teachers' Beliefs and Practices Related to Mathematics Instruction. Teaching and Teacher Education, 17(2), 213-226.

Voss, T., Kleickmann, T., Kunter, M. \& Hachfeld, A. (2011). Überzeugungen von Mathematiklehrkräften. In M. Kunter, J. Baumert, W. Blum, U. Klusmann, S. Krauss \& M. Neubrand (Hrsg.), Professionelle Kompetenz von Lehrkräften. Ergebnisse des Forschungsprogramms COACTIV (S. 235-258). Münster. Waxmann.

Weinert, F. E. (1999). Concepts of Competence: Definition and Selection of Competencies. Munich: Max Planck Institute for Psychological Research.

Weinert, F. E. (2001). Concept of Competence: A Conceptual Clarification. In L. H. Salganik (Hrsg.), Defining and Selecting Key Competencies (S. 45-65). Seattle: Hogrefe \& Huber Publishers.

Wilson, M. \& Cooney, T. (2002). Mathematics Teacher Change and Development. In G. C. Leder, E. Pehkonen \& G. Torner (Hrsg.), Beliefs: A Hidden Variable in Mathematics Education? (S. 127-147). Dordrecht: Kluwer Academic. 